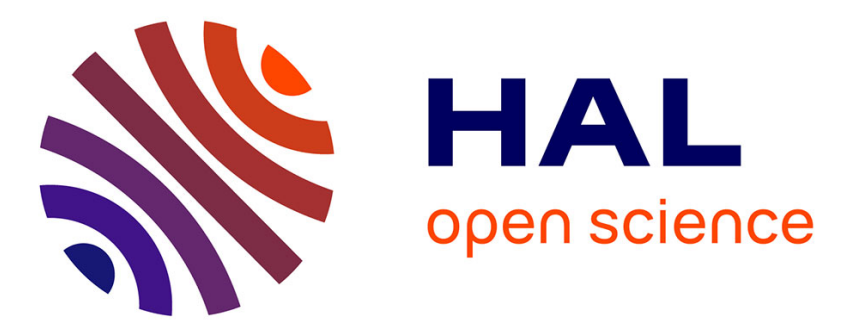

\title{
Perinatal fluoxetine has enduring sexually differentiated effects on neurobehavioral outcomes related to social behaviors
}

Mary Gemmel, Sonsoles de Lacalle, Sophia C Mort, Lesley A Hill, Thierry D

Charlier, Jodi L Pawluski

\section{To cite this version:}

Mary Gemmel, Sonsoles de Lacalle, Sophia C Mort, Lesley A Hill, Thierry D Charlier, et al.. Perinatal fluoxetine has enduring sexually differentiated effects on neurobehavioral outcomes related to social behaviors. Neuropharmacology, 2019, 144, pp.70-81. 10.1016/j.neuropharm.2018.10.009 . hal01903260

\section{HAL Id: hal-01903260 \\ https://hal-univ-rennes1.archives-ouvertes.fr/hal-01903260}

Submitted on 9 Nov 2018

HAL is a multi-disciplinary open access archive for the deposit and dissemination of scientific research documents, whether they are published or not. The documents may come from teaching and research institutions in France or abroad, or from public or private research centers.
L'archive ouverte pluridisciplinaire HAL, est destinée au dépôt et à la diffusion de documents scientifiques de niveau recherche, publiés ou non, émanant des établissements d'enseignement et de recherche français ou étrangers, des laboratoires publics ou privés. 
Perinatal fluoxetine has enduring sexually differentiated effects on neurobehavioral outcomes related to social behaviors.

Mary Gemmel ${ }^{1}$, Sonsoles De Lacalle ${ }^{2}$, Sophia C. Mort ${ }^{2}$, Lesley A. Hill ${ }^{3}$, Thierry D. Charlier ${ }^{4}$ and Jodi L. Pawluski $^{4 *}$

${ }^{1}$ Department of Biological Sciences, Ohio University, Athens, Ohio, USA

${ }^{2}$ Department of Biomedical Sciences, Ohio University, Athens, Ohio, USA

${ }^{3}$ Cellular and Physiological Sciences, Faculty of Medicine, The University of British Columbia, Vancouver, Canada

${ }^{4}$ Univ Rennes, Inserm, EHESP, Irset (Institut de Recherche en Santé, Environnement et Travail), UMR_S 1085, F-35000 Rennes, France

Declarations of interest: JLP has received an honorarium from BINC-Geneva.

*Corresponding Author: Jodi L. Pawluski, Ph.D., IRSET-INSERM U1085, Université de Rennes 1, Campus Villejean, 9 avenue du Prof. Leon Bernard, 35000 Rennes, FRANCE, Phone: $+33(0) 2$ 23.23.41.90, Email : j.pawluski@gmail.com / Jodi-lynn.pawluski@univ-rennes1.fr 


\section{ABSTRACT}

Selective serotonin reuptake inhibitor medications (SSRIs) are prescribed to up to $10 \%$ of pregnant women to treat maternal mood disorders. Exposure to these medications in-utero has raised concerns about altered neurobehavioral outcomes; most recently those related to peer-to-peer social interactions and play. While clinical data show that both perinatal SSRIs (pSSRI) and maternal stress can contribute to social behavioral changes in children, minimal animal work has investigated the effects of pSSRIs in relevant models of maternal stress or the long-term implications of these effects. Therefore the aim of this work was to investigate the long-term effects of pSSRI exposure to fluoxetine on social behaviors, the hypothalamic pituitary adrenal system (HPA) and hippocampal plasticity in adult male and female rat offspring using a model of pre-gestational maternal stress. Adult Sprague-Dawley female and male rat offspring from the following four groups were utilized: 1.Control + Vehicle, 2. Control + Fluoxetine, 3.Pre-gestational Stress + Vehicle, 4. Pre-gestational Stress + Fluoxetine (n=8-16/female/age groups, $\mathrm{n}=8-14 /$ male/age groups). Main findings show pSSRIs increased social investigation in adult females and increased social play (pouncing, nape attacks) in adult males. Perinatal SSRIs also had sexually differentiated effects on hippocampal neurogenesis and GR density. Pre-gestational stress had enduring effects by decreasing social investigation and hippocampal neurogenesis in adult males. Thus pSSRIs, as well as pre-gestational maternal stress, have significant long-term effects on social neurobehavioral outcomes which differ in males and females. This suggests that it would be valuable to consider fetal-sex specific treatments for maternal mental illness.

Keywords: Perinatal Depression; SSRI; social behavior; neurogenesis; sex differences; HPA; play 


\section{Introduction}

Selective Serotonin Reuptake Inhibitor medications (SSRIs) are commonly prescribed during pregnancy to treat maternal stress-related mood disorders (Gemmel et al., 2018a; Oberlander et al., 2006). However, perinatal exposure to these medications (pSSRIs) has been linked to poor social development in children highlighted by increased risk of Autism Spectrum Disorder (ASD) (Man et al., 2015) and attention-deficit hyperactivity disorder (ADHD) (Clements et al., 2015) diagnoses. Children prenatally exposed to SSRIs also exhibit low adaptive and social-emotional behavioral scores, and increased externalizing behaviors such as aggression, hyperactivity, and oppositional/defiant behavior (Gemmel et al., 2018a). However, parental depression and poor maternal health in the year prior to conception play an equally, or even more important, role in childhood behavioral outcomes (Brown et al., 2017; Clements et al., 2015; Mezzacappa et al., 2017). Thus, there is a complex relationship between early life exposure to maternal mood disorders and pSSRIs on the development of social behaviors which requires further investigation.

An increasing body of animal research has aimed to understand the effects of pSSRIs on social behaviors. Early-life SSRI treatment reduces social play behaviors including boxing, wrestling, and following/chasing behaviors in juvenile rats (Khatri et al., 2014; Rodriguez-Porcel et al., 2011) as well as social preference via reduced conspecific/object contacts in both males and females in the juvenile and adult period (Khatri et al., 2014; Rodriguez-Porcel et al., 2011; Simpson et al., 2011). However, previous work focused on paradigms in which offspring were directly treated with SSRIs during the postnatal period and, therefore, neglected to consider perinatal exposure-induced outcomes, or outcomes in the context of relevant models of perinatal affective disorders. With this in mind we have shown that in juvenile offspring, pSSRIs prevent the effects of maternal stress on sibling play, but increase aggressive type social play interactions with a novel same-sex stimulus conspecific (Gemmel et al., 2017). Whether these effects persist into adulthood are not known. Interestingly postnatal exposure to SSRIs can increase proceptive behaviors and sexual receptivity in adult female rat offspring while decreasing male 
copulatory behaviors (Rayen et al., 2013, 2014), suggesting a long-term impact of pSSRIs on social behaviors in general.

Although limited, there is work documenting sexually-differentiated effects of pSSRIs. Apart from the above mentioned effects on copulatory behaviors, early postnatal SSRI treatment reduces juvenile social preference in male, but not female, rat offspring (Simpson et al., 2011) and increases male stereotypic behaviors, such as self-grooming, during social interaction (Rodriguez-Porcel et al., 2011). Developmental SSRIs also increase immobility in the forced swim in adult female rat offspring (Boulle et al., 2016a, b), regardless of exposure to maternal stress. However, in the absence of a maternal stress paradigm, perinatal SSRI exposure can also increase anxiety and depressive-like behaviors in adult male rodent offspring (Ansorge et al., 2004; Oberlander et al., 2009). It remains to be determined how pSSRIs, in the presence of maternal stress, affect peer-to-peer social interactions in adult male and female offspring.

Perinatal SSRIs also alter the developing hypothalamic-pituitary-adrenal axis (HPA) (Avitsur, 2017; Avitsur et al., 2016; Pawluski et al., 2012b), a system that has been linked to social interactions and aggressive phenotypes (Walker et al., 2016). For example, unusually low or high HPA axis reactivity is found with the emergence of aggressive behaviors. Work in animal models has shown that pSSRIs have a long-term impact on the HPA system by decreasing corticosterone levels, increasing corticosteroid binding globulin (CBG) levels, altering glucocorticoid receptor density in the hippocampus of offspring, and normalizing the effects of maternal stress on density of dendritic spines and synapses in the hippocampal CA3 region (Gemmel et al., 2017; Ishiwata et al., 2005; Pawluski et al., 2012b). More recently, research shows that pSSRIs, in the absence of maternal stress, alter the ability to respond to continuous stress in both male and female mice offspring (Avitsur, 2017; Avitsur et al., 2016). How these changes in the HPA axis are related to pSSRI effects on social behaviors remains to be determined.

Research has also highlighted the role of the hippocampus in HPA function as well as the formation of social memories pointing to an important role of the CA2 region in this regard (Hammels et 
al., 2015; Hitti and Siegelbaum, 2014; Stevenson and Caldwell, 2014). Thus, changes in social behaviors as a result of pSSRIs are likely related to neurobiological changes within this critical limbic region. In pre-adolescent offspring, there was a significant correlation between social interaction with a novel conspecific and the density of glucocorticoid receptors (GR), as well as, pre- and post- synaptic proteins in the CA2 region with additional effects of pSSRIs and pre-gestational maternal stress on neurogenesis and plasticity (Gemmel et al., 2017). Interestingly, the relationship between social interactions and plasticity measures in the CA2 are more pronounced in pre-adolescent female offspring (Gemmel et al., 2017) but it remains to be determined whether these effects endure into adulthood.

The present study aimed to determine the persistent effect of perinatal SSRI exposure to fluoxetine, a popular SSRI prescribed to pregnant women, on social interactions and related changes in the HPA axis and hippocampal plasticity in adult male and female offspring. To determine how changes in social behaviors with a novel same-sex conspecific were linked to the HPA axis, serum levels of corticosterone after social interaction, as well as serum levels of CBG and GR density in the hippocampus and periventricular nucleus (PVN), were assessed. Measures of hippocampal plasticity were also investigated by measuring synaptic proteins in the CA2, CA3, and dentate gyrus and markers of adult neurogenesis in the granule cell layer. A pre-gestational maternal stress paradigm was used as a history of depressive-episodes, anxiety, or prolonged stress prior to pregnancy increase risk of perinatal depression and anxiety (Stewart, 2011). Previous work in animal models shows that pre-gestational chronic unpredictable stress for three weeks immediately prior to gestation can affect both the mother and offspring. For example, pre-gestational stress can affect the developing 5HT system as well as HPA axis reactivity in rat offspring (Gemmel et al., 2017; Gemmel et al., 2018c; Huang et al., 2013; Huang et al., 2010; Huang et al., 2012). Expanding on previous work we expected that pSSRIs would increase social interactions in female offspring, while pre-gestational maternal stress would decrease social behaviors in male offspring, with the exception of social play (pouncing/nape attacks) which has been linked to serotonin levels (Kepser and Homberg, 2015; Kiryanova and Dyck, 2014). We predicted that pSSRIinduced effects on social interactions would be related to alterations in the HPA system as well as the 
CA2 region of the hippocampus. Understanding the long-term impact of pSSRIs, in a model of maternal stress/depression, on social interactions and related neuroendocrinology in both male and female offspring is needed to understand the benefits and risks of exposure to antidepressant medications during the perinatal period.

\section{Materials and methods}

2.1. Animals. Prior to breeding, thirty-four adult female (175-199 g, approximately 60 days of age) and nine adult male (275-299 g) Sprague-Dawley rats from Harlan Laboratories Inc. (Indianapolis, Indiana) were kept in a 12:12-h light/dark schedule under standard laboratory conditions. All experiments were approved by the Institutional Animal Care and Use Committee of Ohio University (IACUC, 12-H-053, 14-H-011) in accordance with the National Institutes of Health guide for the care and use of Laboratory animals (NIH Publications No. 8023, revised 1978).

Three weeks prior to breeding, females were assigned randomly to stress or control groups (16 control, 18 stress). Stressed females were individually housed and subjected to three weeks of chronic unpredictable stress (CUS) consisting of 0-2 stressors per day (restraint stress under bright light, cage rotation, overcrowding, food depravation, wet bedding, forced swim) as previously described (Gemmel et al., 2017) to model aspects of depression and anxiety. For a detailed table of stressors used in the present study see Table 1 . Following the 3-week CUS cessation, one female and one male were housed together and gestation day (GD) 1 was identified by a vaginal smear and evidence of sperm in the vagina. Following GD1, all dams were housed individually and monitored daily. The final number of dams that remained pregnant until term were $24(\mathrm{CV}=6, \mathrm{CF}=8, \mathrm{PGSV}=5, \mathrm{PGSF}=5)$. We have recently shown that this stress paradigm resulted in decreased maternal weight gain, poor maintenance of pregnancy, and altered corticosterone levels in these rat dams (Gemmel et al., 2018b).

2.2. Fluoxetine administration to dams. Fluoxetine (Fagron, Belgium) or vehicle was administered orally via a wafer biscuit from GD10 until weaning (postnatal day (PD) 21). The dams received $1 / 8^{\text {th }}$ of the 
wafer biscuit twice a day filled with vehicle (saline), or $5 \mathrm{mg} / \mathrm{kg}$ of fluoxetine in vehicle, as described (Gemmel et al., 2017) for a total dose of $10 \mathrm{mg} / \mathrm{kg} /$ day of fluoxetine. To ensure the dam, and not offspring, consumed the entire biscuit, wafer feeding was completed under supervision.

2.3. Maternal Caregiving Behaviors. To assess any overall effects of maternal care on behavioral outcomes in offspring, maternal caregiving behaviors of offspring were observed in the first week postpartum (PD 1-6), twice per day for 5 min. (Gemmel et al., 2017). Duration of the following maternal behaviors were assessed, and data aggregated across days: licking (licking/grooming), and nursing (arched-back nursing, blanket nursing or passive nursing) to control for any gross effects of maternal care-giving on offspring outcomes. Specific maternal care-giving details in these dams has been recently published and results show that pre-gestationally stressed dams showed higher levels of nursing and fewer bouts of licking/grooming offspring in the first week postpartum (Gemmel et al., 2018b).

2.4. Adult offspring. On PD 1, litters were culled to 4 males and 4 females. Offspring were housed with their mothers until weaning (PD21), then housed with same-sex littermates (4 per cage) and housed 2 per cage at 30 days of age. During adulthood ( $7-8^{\text {th }}$ postnatal week), a maximum of 2 male and 2 female offspring from a total of 24 litters were used to minimize litter-effects. Offspring from the following treatment groups were used: 1) Control+Vehicle (CV; 10 females, 12 males), 2) Control+Fluoxetine (CF; 14 females, 16 males), 3) Pre-gestational Stress+Vehicle (PGSV; 8 females, 8 males), and 4) Pregestational Stress+Fluoxetine (PGSF; 9 females and 10 males).

2.5. Social Interaction Test (SI). Social interaction testing to assess social investigation and play behaviors was completed $24 \mathrm{~h}$ following a $15 \mathrm{~min}$ habituation to the apparatus during the lights-on portion of the light cycle (between 0900 and 1300). Each experimental animal was paired with a sex- and weight-matched non-experimental stimulus animal bred at our facility specifically to serve as a stimulus partner. A total of 6 male and 6 female stimulus rats were used from 3 litters. These animals were 
habituated to the chamber for $10 \mathrm{~min}$ prior to testing and were not naïve to social interaction prior to testing with experimental animals.

Sessions were recorded by an overhead digital camera, and videos were evaluated by a blinded observer based on previous work (Crawley, 2012; van Kerkhof et al., 2013; Veenema, 2012). For each experimental animal the duration (s) of the following behaviors were scored: sniffing the stimulus rat; following the stimulus rat; crawling over or under the stimulus rat; grooming the stimulus rat; pouncing on the stimulus rat (experimental rat soliciting the stimulus rat); pinning (the experimental rat holds the stimulus rat on its back); nape attack (the experimental animal displaying nose attacks or nose contacts toward the nape of the neck of the stimulus animal); and running away from the stimulus rat; Selfgrooming in the experimental rat was also assessed. Following and sniffing were combined to a single 'social investigation' variable based on previous research (Crawley, 2012; Veenema et al., 2012). In addition, social play behaviors such as pinning, pouncing, and nape-attacks were combined to a single 'social play' variable based on previous work (Veenema et al., 2012). Time until first interaction, total time in each scored behavior, and time interacting were recorded for the experimental animal and can be found in Table 3.

2.6. Corticosterone response to SI. Tail nicks were performed immediately following social interaction testing to obtain blood samples for assessment of corticosterone response to social interaction with a novel conspecific.

2.7. Corticosterone binding globulin (CBG) capacity. At euthanasia (within $24 \mathrm{~h}$ of behavioral tests - see below), blood samples were collected and centrifuged at 10,000×g for $10 \mathrm{~min}$, serum separated, and stored at $-80{ }^{\circ} \mathrm{C}$. To assess the binding capacity of $\mathrm{CBG}$, samples were diluted 1/1000 for males and 1:1500 for females and incubated with dextran-coated charcoal (DCC) to remove endogenous steroids for $30 \mathrm{~min}$ at room temperature. Centrifugation was used to sediment DCC, and $100 \mu 1$ aliquots were dispensed into duplicate tubes containing $\left[1,2-{ }^{3} \mathrm{H}\right]$ corticosterone (specific activity: $50 \mathrm{Ci} / \mathrm{mmol}$; ARC, St. 
Louis, MO, USA, final concentration $\sim 10 \mathrm{nM}$ ) and another tube containing $1 \mathrm{mM}$ cold corticosterone to evaluate non-specific binding. Samples were incubated at room temperature for $1 \mathrm{~h}$, followed by an ice water bath for $30 \mathrm{~min}$. Ice-cold DCC was added for $10 \mathrm{~min}$, and tubes centrifuged at $1800 \mathrm{~g}$ for $10 \mathrm{~min}$ at $4{ }^{\circ} \mathrm{C}$ to remove unbound steroids. To account for the dissociation of $\left[{ }^{3} \mathrm{H}\right]$ corticosterone from CBG during the latter DCC separation step, a correction factor was used as described (Hammond and Lahteenmaki, 1983). Supernatants were transferred into scintillation vials with $4 \mathrm{ml}$ of Aqueous Counting Scintillant (PerkinElmer, Waltham, MA, USA) to determine the CBG-bound $\left[{ }^{3} \mathrm{H}\right]$ corticosterone after subtracting non-specifically bound $\left[{ }^{3} \mathrm{H}\right]$ corticosterone and using this to determine the corticosterone binding capacity of CBG in nM. Inter-assay variability was $5.1 \%$. Estimates of free corticosterone were computed with the equation total corticosterone $(\mathrm{nM}) * 100 / \mathrm{CBG}(\mathrm{nM})$ (FCI - free corticosterone index) as previously described (Pawluski et al., 2012a).

2.8. Histology. Within $24 \mathrm{~h}$ of SI testing, adult offspring were euthanized (between 1100 and 1500) by deep anesthetization with isofluorane and decapitation. Brains were rapidly dissected, immersion fixed in $4 \%$ paraformaldehyde for $48 \mathrm{~h}$, and then placed in saturated $30 \%$ sucrose solution for approximately a week. Brains were stored at $-80^{\circ} \mathrm{C}$ until coronally sliced by cryostat (Leica Biosystems, Wetzlar, Germany) in 40 micron sections in series of 12. Tissue sections were transferred to a glycol based antifreeze solution and stored at $-20^{\circ} \mathrm{C}$.

For immunohistochemistry, dorsal hippocampal sections (bregma -2.64 to -4.92) from a random sample of 6 animals per sex per group was used to assess synaptic proteins (PSD-95, synaptophysin) and the glucocorticoid receptor (GR) expression in the CA2, CA3, and dentate gyrus (DG) regions, as well as immature neurons (doublecortin/DCX) in the granule cell layer of the dorsal DG as described (Gemmel et al., 2017). Sections were rinsed between steps in PBS and PBS plus $0.01 \%$ Triton X-100 (PBST) for GR staining or TBS and TBS plus $0.01 \%$ Triton X-100 (TBST) for all other staining. Tissue was incubated for $30 \mathrm{~min}$ at room temperature in $0.6 \% \mathrm{H}_{2} \mathrm{O}_{2}$ followed by blocking for $30 \mathrm{~min}$ at room temperature in $5 \%$ Normal Goat Serum in PBST or TBST (for GR, synaptophysin, and PSD-95 staining; Lampire Biological 
Laboratories, Pipersville PA, USA). For PSD-95 staining, an additional antigen unmasking step was completed for $20 \mathrm{~min}$ at $80^{\circ} \mathrm{C}$ using $10 \mathrm{mM}$ sodium citrate buffer ( $\mathrm{pH}$ 6.0) prior to incubation with $\mathrm{H}_{2} \mathrm{O}_{2}$. Tissue was then incubated overnight at $4^{\circ} \mathrm{C}$ in primary goat anti-doublecortin (1:200, Santa Cruz Biotechnology, Dallas Texas, USA), mouse anti-synaptophysin (1:500, Sigma Aldrich, St. Louis MO, USA: NGS+TBST) or rabbit anti-PSD-95 antibody (1:1000, Abcam, Cambridge, MA, USA: NGS+TBST), or in rabbit anti-GR antibody (1:500, Abcam, Cambridge, MA, USA) for two nights. Sections were incubated in appropriate secondary antibody (Vector Laboratories, Burlingame, CA, USA) at room temperature for $2 \mathrm{~h}$ followed by processing using the avidin-biotin complex (ABC Elite kit; 1:500; Vector laboratories, Burlingame, CA, USA) and DAB (3,3-diaminobenzidine; Vector laboratories, Burlingame, CA, USA). Sections were mounted on Superfrost Plus slides (Fischer Scientific, Pittsburgh, PA), dried, dehydrated, and cover-slipped with Permount (Fischer Scientific, Pittsburgh, PA). For a representative photomicrograph see Figure 1.

2.9. Quantification. The number of DCX immunoreactive (-ir) cells were counted bilaterally throughout the dorsal GCL/SGZ in 1 series of brain sections (on every $12^{\text {th }}$ hippocampal section) under a $40 \mathrm{x}$ objective, as previously described (Barker and Galea, 2008; Gemmel et al., 2017; Gemmel et al., 2015; Gemmel et al., 2016b; Rayen et al., 2015). Cavalieri's principle was used to estimate the total number of DCX-ir cells in the dorsal hippocampus. For optical densities of GR, synaptophysin, and PSD-95-ir cells, two photomicrographs in were taken of each CA2, CA3, and DG/GCL/SGZ region in two sections of the dorsal hippocampus at similar locations between stereotaxic coordinates $-2.64 \mathrm{~mm}$ to $-4.92 \mathrm{~mm}$ bregma based on previous work (Gemmel et al., 2017; Gemmel et al., 2015; Pawluski et al., 2012a; Rayen et al., 2015). Photomicrographs for all sections were taken at 40x objective using a Nikon Microphot SA and Nikon DS-Qi1MC camera with Nikon NIS Elements F4.00 software and quantified using Image J software (Wayne Rasband, NIH, Bethesda MD, USA). Two photomicrographs of GR-immunoreactive cells were also quantified for optical density in the paraventricular nucleus (PVN) of the hypothalamus (bregma -1.72 to -1.92). For PVN analysis, photomicrographs were examined under a 40x objective using 
(Olympus Provis, equipped with a DP71 digital camera, program Cell ^p) and quantified using Image J64 software as stated above. Quantification was completed by a researcher blind to the conditions.

2.10. Steroid hormones analysis. Blood samples were centrifuged at $10,000 \times \mathrm{g}$ for $10 \mathrm{~min}$, serum collected, and stored at $-80{ }^{\circ} \mathrm{C}$ until analysis. For corticosterone levels after SI, samples were run in duplicate using a RIA kit for rat corticosterone from MP Biomedicals (Corticosterone I125 for rats and mice, MP Biomedicals, Santa Ana, CA, USA). The assay had a sensitivity of 7.7 ng and the average coefficient of variation was less than $5 \%$. Corticosterone levels were converted to $\mathrm{nM}$ for consistency with the CBG results below by using the equation $\mathrm{ng} / \mathrm{ml} * 3.46$.

Quantitative determination of serum estradiol in adult female offspring and serum testosterone in adult male offspring (at euthanasia) were completed by enzyme-linked immunosorbent assay following predetermined kit instructions (ALPCO Estradiol Rat ELISA 55-ESTRT-E01, or ALPCO Testosterone Rat/Mouse ELISA 55-TESMS-E01, ALPCO Diagnostics, Salem, NH, USA), with all samples being run in duplicate. Sensitivity of the ELISA was f $2.5 \mathrm{pg} / \mathrm{mL}$ for estradiol and $3.06 \mathrm{ng} / \mathrm{mL}$ for testosterone. The average coefficient of variation was less than $7 \%$.

2.11. Statistical Analysis. Data were analyzed using the software Statistica (Dell Inc.). Due to previous findings of sexually differentiated patterns of outcomes in the response to pSSRIs and maternal stress exposure on behavioral measures, HPA axis, hippocampal plasticity, and additional neurobehavioral outcomes (Gemmel et al., 2018a; Gobinath et al., 2016; Rayen et al., 2015), factorial ANOVAs were conducted separately for each sex on behavioral measures, corticosterone, CBG, as well as on measures of hippocampal plasticity and GR density with condition (pre-gestational stress/control) and treatment (fluoxetine/vehicle) as independent factors. Factorial ANOVAs with condition (pre-gestational stress/control), treatment (fluoxetine/vehicle), and sex (male/female) as independent factors can be found in Table 2. Significant interaction effects were analyzed by a Fisher LSD post hoc test to compare individual group differences. Pearson correlations were analyzed between maternal caregiving behaviors, 
corticosterone and CBG measures, social behaviors and neural measurements. Any effects of maternal care-giving behavior or gonadal hormones (estradiol in females and testosterone in males) were also controlled for by using analysis of covariance (ANCOVA) tests. Significance was set at $p<0.05$.

\section{Results}

3.1. Social Interaction Test. Analysis by sex of social behaviors revealed different patterns of effects in females and males. In adult female offspring, there was a significant main effect of perinatal fluoxetine on time in novel partner-directed social behaviors $(\mathrm{F}(1,37)=5.94, \mathrm{p}=0.02$, Figure $2 \mathrm{~A})$ with perinatal fluoxetine exposed females spending more time interacting with a stimulus female. When looking at specific behaviors, perinatal fluoxetine exposed females spent significantly more time engaged in social investigation (main effect of treatment; $\mathrm{F}(1,37)=12.34, \mathrm{p}=0.002$, Figure $2 \mathrm{~B}$ ). There was also a significant effect of perinatal fluoxetine exposure on social grooming (main effect of treatment; $F(1,37)=4.38$, $\mathrm{p}=0.04$, Table 3). A significant condition by treatment effect was found with time to first interaction ( $\mathrm{F}(1,37=6.19, \mathrm{p}=0.02$, Table 3$)$ with PGSV females taking significantly longer to be socially active compared to CV ( $\mathrm{p}=0.04)$ and PGSF $(\mathrm{p}=0.001)$ females, while CV females took longer to be socially active compared to PGSF females $(\mathrm{p}=0.04)$. There was also a significant main effect of treatment on time to first interaction $(\mathrm{F}(1,37)=8.74, \mathrm{p}=0.005)$, with fluoxetine exposure reducing time to first interaction in females. There were no significant differences in social play in adult females (Figure 2C) and no other significant differences in social behaviors of adult female offspring.

In adult male offspring, there was a significant main effect of condition on time in novel partnerdirected behaviors $(F(1,42)=6.05, p=0.02$, Figure $2 \mathrm{D})$ with males exposed to pre-gestational maternal stress spending less time interacting with a novel stimulus partner. When looking at specific social behaviors, pre-gestationally stressed males spent significantly less time in social investigation with a novel partner compared to control males (main effect of condition; $F(1,42)=18.24$, $p=0.0001$, Figure 2E). In adult male offspring there was also a significant main effect of perinatal fluoxetine treatment on time in 
social play $(F(1,42)=4.49, p=0.04$, Figure $2 F)$ with males exposed to perinatal fluoxetine spending significantly more time in social play than vehicle exposed offspring. Perinatal fluoxetine exposure significantly affected running away from a novel partner (main effect of treatment: $F(1,42)=10.63$, $\mathrm{p}=0.002$, Table 3$)$ and self-grooming $(\mathrm{F}(1,42)=5.37, \mathrm{p}=0.03$, Table 3$)$, with fluoxetine exposed males running away more often and self-grooming more often than vehicle exposed males.

There were no other significant main effects, interaction effects, (Table 3) or correlations and no effect of maternal care-giving or gonadal hormone levels on behavioral outcomes in females or males (ANCOVA, $0.06<\mathrm{p}<0.9$ ).

3.2. HPA Outcomes. In the hippocampus of adult females, there was a significant main effect of treatment $(\mathrm{F}(1,20)=5.94, \mathrm{p}=0.02$, Figure $3 \mathrm{~A})$ on glucocorticoid receptor density in dentate gyrus, with females exposed to fluoxetine having significantly greater GR density compared to vehicle exposed females. There was also a main effect of condition $(\mathrm{F}(1,20)=5.01, \mathrm{p}=0.04$, Figure $3 \mathrm{~A})$ on GR density in the dentate gyrus, with adult females exposed to pre-gestational maternal stress having significantly lower density in this region compared to control females. Controlling for adult female estradiol levels diminished the effect of pre-gestational maternal stress on GR density in the DG (ANCOVA; effect of condition; $\mathrm{F}(1,20)=2.36, \mathrm{p}=0.1)$. There were no other significant effects of condition or treatment on GR density in the hippocampus (Figure 3B, Table 4) or significant correlations between GR density and social behaviors of female offspring (Figure 3C).

In the hippocampus of adult male offspring, there were no significant effects on GR density in the DG, as evident in the female offspring (Figure 3D), but there was a significant main effect of treatment $(F(1,20)=4.38, p=0.049$, Figure $3 E)$ on GR density in the CA3 region, with males perinatally exposed to fluoxetine having significantly reduced GR density compared to vehicle exposed males. There was also a significant positive correlation between GR density in the DG of adult males and total time in interacting with a novel conspecific $(\mathrm{r}=0.54, \mathrm{p}=0.01$, Figure $3 \mathrm{~F})$. There were no other significant correlations between GR density, social behaviors or maternal care-giving behaviors in male or female offspring. 
In adult females there was a significant main effect of pre-gestational maternal stress on serum corticosterone levels after social interaction $(F(1,34)=4.24, p=0.04$, Table 4) and on $C B G(F(1,37)=5.58$, $\mathrm{p}=0.02$, Table 4) with adult females exposed to pre-gestational maternal stress having significantly lower corticosterone levels and lower CBG compared to control females. Controlling for estradiol levels diminished the effect of pre-gestational maternal stress on serum corticosterone (ANCOVA; effect of condition; $\mathrm{F}(1,34)=2.87, \mathrm{p}=0.09)$.

There were no additional significant effects on free corticosterone, corticosterone or CBG measures in females or males (Table 4) ( $p>0.09$ ). There was no effect of maternal care-giving on HPA measures in females or males (ANCOVA, $0.06<\mathrm{p}<1.0$ ).

3.3. Hippocampal Plasticity. In adult females, there was a significant main effect of treatment on total number of immature neurons in the hippocampal GCL/SGZ $(F(1,20)=4.60, p=0.04$, Figure 4A) with perinatal fluoxetine exposure significantly increasing doublecortin-ir. There was also a significant main effect of treatment on post-synaptic density via PSD-95-ir in the CA2 region in adult female offspring $(\mathrm{F}(1,20)=5.31, \mathrm{p}=0.03$, Figure 4B) with perinatal fluoxetine exposure significantly reducing post-synaptic density. There was a non-significant effect of treatment on post-synaptic density in the dentate gyrus ( $\mathrm{p}=0.06$, Table 5). ANCOVAs revealed no significant effects of estradiol levels on any of the measures $(\mathrm{p}>0.6)$.

In adult male offspring, there was a significant main effect of condition on total number of immature neurons in the hippocampal GCL/SGZ $(\mathrm{F}(1,20)=8.32$, $\mathrm{p}=0.009$, Figure 4C) with male offspring exposed to pre-gestational maternal stress having significantly less doublecortin-ir. There was also a significant main effect of treatment on post-synaptic density via PSD-95-ir in the CA2 in adult male offspring $(F(1,20)=6.79, p=0.02$, Figure $4 D)$ with perinatal fluoxetine exposure significantly reducing post-synaptic density. There was a significant positive correlation between testosterone levels and synaptophysin density in the dentate gyrus of adult males $(r=0.44, p=0.04$, Supplementary Figure 1). There were no other significant differences in adult males or females. There was no effect of maternal care-giving on neural measures in females or males (ANCOVA, $0.09<\mathrm{p}<0.96$ ). 


\section{Discussion}

The current work highlights persistent effects of perinatal fluoxetine exposure, as well as pregestational stress, on social behaviors, the HPA axis, and hippocampus plasticity that are sexually differentiated in adulthood. Main findings show that perinatal fluoxetine increased social investigation in adult female offspring and increased social play in adult male offspring. Different patterns of outcomes in male and female offspring, resulting from perinatal fluoxetine exposure, persisted in the hippocampus with regards to GR density and neurogenesis.

\subsection{Perinatal SSRIs, Pre-gestational Stress, and Social Behaviors}

In the present study perinatal fluoxetine increased social investigation in adult female offspring. Overall, adult females perinatally exposed to fluoxetine were quicker to interact with a novel same-sex conspecific and spent more time engaged in interactions with a novel conspecific. Previous work has shown that perinatal fluoxetine exposure increases female conspecific interaction from postnatal day 2831 (Svirsky et al., 2016) and facilitates female rat sexual behavior by a significant increase in proceptive behaviors, the lordosis quotient, and decrease in time spent rejecting male advances (Rayen et al., 2014). Interestingly, in pre-adolescent females we found that perinatal fluoxetine did not increase social investigation (Gemmel et al., 2017). This transition to 'pro-social' behaviors in adult female offspring is likely dependent on both organizational effects of pSSRIs and activational effects of gonadal hormones after puberty (Dohler et al., 1991). These 'pro-social' behaviors may be beneficial by increasing social bonds, improving social networks, and ensuring reproductive success. However, it should be noted that clinical work, and animal models, show that pSSRIs are also associated with increased anxiety and depressive-like phenotypes in both males and females (Boulle et al., 2016a; Malm et al., 2016; Olivier et al., 2013).

In addition to effects in adult females, our results show that perinatal fluoxetine exposure increased social play (pinning, nape/attacks), running away, and self-grooming in adult male offspring 
when paired with a novel same-sex stimulus conspecific. This expands our previous work in preadolescent male offspring which shows that perinatal fluoxetine exposure increases social play (Gemmel et al., 2017). Although perinatal exposure to SSRIs in relation to social play is less well studied, increases in serotonin due to the absence of the serotonin transporter or acute administration of fluoxetine has resulted in reductions in social play during adolescence, at least in males (Homberg et al 2007)(Kiser et al., 2012). Interestingly perinatal fluoxetine exposure has the opposite effect and it may be that altering the central serotonergic system with SSRIs during development alters neural circuitry related to social play phenotypes and contributes to increased social play in male rat offspring throughout life.

Pre-gestational stress also had enduring effects on social behaviors in adult male offspring resulting in significantly less social investigation and less time interacting with a novel same-sex conspecific. Therefore, both pSSRIs and pre-gestational maternal stress have enduring and behaviorally distinct effects on social behaviors in adult male offspring. Although these behavioral phenotypes have not been investigated in the clinical population, this work does suggest that there is a link between pSSRIs and/or maternal mental illness and specific behavioral outcomes. Regardless, this work is in agreement with clinical work showing that both pSSRIs and maternal mental illness can have enduring effects on social behaviors in children (Brown et al., 2017; Man et al., 2015; Mezzacappa et al., 2017; Oberlander and Zwaigenbaum, 2017). For example, maternal mood symptoms and pSSRIs are related to increased internalising behaviors in 3 and 4 year old children (Oberlander et al., 2010). pSSRIs have a history of affecting early behavioral development, even when controlling for maternal mental illness: Infants exposed to SSRIs during gestation exhibit blunted emotional and physical response to painful stimuli at two months of age, low adaptive and social-emotional behavioral scores at 10 months of age (Hanley et al., 2013; Oberlander et al., 2002; Oberlander et al., 2005), increased scores on the Pervasive Developmental Disorder scale at 2-5 years of age (Johnson et al., 2016), and increased externalizing behavior at 4 years of age (Hermansen et al., 2016; Oberlander et al., 2007). However, it must be acknowledged that the underlying state of maternal mental health and stress, as well as additional 
environmental factors (Park and Oberlander, 2018), can mediate or moderate these pSSRI effects. Thus, the present work expands clinical work showing that pSSRIs and maternal depression may both contribute to developing social behaviors, particularly in male offspring.

That being said, findings from the present study show that pSSRIs and pre-gestational maternal stress have distinct effects on behavioral outcomes in adult male and female offspring. For example, pSSRIs increase social investigation in adult female offspring, while increasing social play in adult male offspring. Furthermore, pre-gestational maternal stress has little effect on social behaviors in female offspring, but decreases social investigation in adult male offspring. These sex differences may not be surprising as it is well documented in animal literature that social behaviors are sexually differentiated with male rodents, being more likely to engage in social play interactions while females being more likely to engage in social investigation and contact behaviors(Taylor et al., 2000). Therefore, pre-disposition toward specific behaviors, as well as natural variation in the frequency of male versus female social behaviors, may play a role in mediating the sex-dependent behavioral outcomes after pSSRIs or pregestational maternal stress.

While there are no definitive rodent behavioral tests which completely mimics human social behaviors, these findings show that perinatal, and pre-gestational factors, can have distinct and enduring patterns of effects on social behaviors that are sexually differentiated. Thus, further work on pSSRIs as treatment for maternal mood disorders should consider the role that fetal sex and child sex and/or gender may play on observed outcomes. In line with this, a recent meta-analysis addressing the relationship between pSSRIs and developmental disorders reported significant relationships with pSSRIs only when addressing male outcomes, or when male bias is included in the sample size (4:1 male to female ratios) (Harrington et al., 2014; Man et al., 2015). Therefore, human work associating pSSRIs to social behaviors needs further incorporation of both male and female offspring (Clements et al., 2015; El Marroun et al., 2014). 
It is important to note that main effects of perinatal fluoxetine or pre-gestational maternal stress were evident in adult offspring but few, if any, interactions between the two factors existed. We have previously shown that pSSRIs can normalize neurobehavioral outcomes in juvenile and adolescent male and female offspring, but this is not the case in adulthood. For example, perinatal fluoxetine exposure prevents the effects of pre-gestational stress on sibling play in pre-adolescent offspring, but the same effect is not seen in adult offspring (Gemmel et al., 2017; Rayen et al., 2015). This suggests that pregestational maternal stress and pSSRIs have distinct neurobehavioral effects in adulthood, as discussed above; pSSRIs are less effective in ameliorating effects of maternal stress in the long term, particularly in male offspring; and gonadal hormones of puberty play a significant role in the long-term effects of pSSRIs and maternal stress, particularly in adult female offspring.

\subsection{Perinatal SSRIs, Pre-gestational Stress, and HPA Outcomes}

In the present study, perinatal fluoxetine affect GR density in the dentate gyrus of adult females and GR density in the CA3 of adult males. Perinatal SSRIs affect the serotonergic system (Gemmel et al., 2016a; Huang et al., 2012; Oberlander et al., 2009) and serotonin can act to increase the density of hippocampal glucocorticoid receptors (Erdeljan et al., 2001, 2005) (Meaney et al., 1994) (Laplante et al., 2002). Therefore, pSSRI exposure, which alter serotonin levels in the fetus, have long-term effects in modulating the density of hippocampal glucocorticoid receptors which likely affects a number of behavioral outcomes. In line with this we found a positive correlation between the GR density in the dentate gyrus and the time spent interacting with a novel same-sex conspecific in adult male, but not female, offspring. This suggests that HPA responsivity may be more precisely regulated as a function of increased GR density and thus make interacting with a novel partner less adversive, particularly in adult males after pSSRI exposure.

\subsection{Perinatal SSRIs, Pre-gestational Stress, and Plasticity in the Hippocampus}

In the present study we found a significant effect of perinatal fluoxetine exposure in reducing post-synaptic density in the CA2 region of the hippocampus in both adult male and female offspring. We 
have also found that, during pre-adolscence, time interacting with a novel conspecific was significantly negatively correlated with the density of both pre- and post- synaptic proteins (Gemmel et al., 2017). Therefore, reductions in post synaptic density in the CA2 region of the hippocampus may play a role in increasing specific social interactions and, perhaps, social memory in adult offspring following pSSRI exposure.

When looking at patterns of hippocampal neurogenesis, we found that perinatal fluoxetine increased neurogenesis in the granule cell layer of adult female offspring. This finding supports and expands our previous work showing that early postnatal fluoxetine exposure increases new cell survival in adult female offspring (Rayen et al., 2015). In adult male offspring, there were no enduring effects of pSSRIs on hippocampal neurogenesis, but pre-gestational maternal stress reduced neurogenesis in the granule cell layer. Much research has shown that prenatal stress decreases hippocampal neurogenesis in adult male, but not female, offspring (Loi et al., 2014; Rayen et al., 2015), but this is the first to show that stress prior to gestation can have these enduring effects in adult male offspring. This also expands our previous work in pre-adolescent offspring showing that pre-gestational maternal stress, regardlesss of perinatal fluoxetine exposure, significantly reduced neurogenesis in the granule cell layer of males, but not females (Gemmel et al., 2017). Taken together this work suggests that neurogenesis in the hippocampal dentate gyrus may contribute to the sex-specific behavioral outcomes observed in the present study, with females being more sensitive to perinatal fluoxetine exposure and males being more sensitive to pre-gestational maternal stress.

\subsection{Conclusions}

The current work demonstrates clear effects of perinatal exposure to the SSRI fluoxetine on social investigation, social play, and neuroplasticity in adult male and female offspring. This works points to a need to consider the sex specific effects of perinatal SSRIs and maternal mood disorders on 
neurobehavioral outcomes in children. Furthermore this work suggests that fetal-sex specific treatments, in general, may be warranted. 


\section{FIGURE LEGENDS}

Figure 1. Representative photomicrographs of A) doublecortin in the GCL, B) synaptophysin-ir in the CA3, C) PSD-95-ir in the CA2, and D) GR-ir cells in the dentate gyrus of control female offspring at 40x objective. (Scale bar is set at $25 \mu \mathrm{m}$ )

Figure 2. Mean (+SEM) time in social behaviors during social interaction in adult female and male offspring (s). A, D) time interacting with a stimulus animal; B, E) time in social investigation; C, F) time in social play. $*$ denotes significance, $\wedge$ denotes significant main effect of treatment $(\mathrm{p}<0.05) . \mathrm{CV}=$ control + vehicle, $\mathrm{CF}=$ control + fluoxetine, $\mathrm{PGSV}=$ pre-gestational stress + vehicle, $\mathrm{PGSF}=$ pregestational stress + fluoxetine.

Figure 3. Measures of HPA outcomes. Mean $( \pm$ SEM) GR density (OD) in the DG (A, D) and CA3 (B, E) of adult female and male offspring. C, F) Correlations between time in social interaction with a novel conspecific and GR density in the dentate gyrus of female and male offspring. *denotes significance, ^ denotes significant main effect of treatment $(\mathrm{p}<0.05) . \mathrm{CV}=$ control + vehicle, $\mathrm{CF}=$ control + fluoxetine, PGSV $=$ pre-gestational stress + vehicle, $P G S F=$ pre-gestational stress + fluoxetine. $(n=6-16 /$ sex/group $)$

Figure 4. Mean $( \pm$ SEM) of $(\mathrm{A}, \mathrm{C})$ immature neurons (DCX-ir cells/slice) in the dorsal GCL and $(\mathrm{B}, \mathrm{D})$ PSD-95-ir in the CA2 region of the hippocampus. *denotes significance, ${ }^{\wedge}$ denotes significant main effect of treatment $(\mathrm{p}<0.05) . \mathrm{CV}=$ control + vehicle, $\mathrm{CF}=$ control + fluoxetine, $\mathrm{PGSV}=$ pre-gestational stress + vehicle, $\mathrm{PGSF}=$ pre-gestational stress + fluoxetine. $(\mathrm{n}=6 / \mathrm{sex} /$ group $)$. 
Acknowledgements. We thank Geoffrey L. Hammond and Eszter Bögi for their invaluable assistance with this work. MG is presently a Postdoctoral Associate at Magee-Womens Research Institute Department of Obstetrics, Gynecology \& Reproductive Sciences, University of Pittsburgh School of Medicine.

Funding: JLP was funded by a Brain \& Behavior Research Foundation NARSAD Young Investigator Grant. JLP and TDC were funded by the Region Bretagne (SAD). TDC is funded by Rennes Metropoles and INSERM. MG received the 2015-2016 Student Enhancement Award from the CRSCA and the 20172018 Donald Clippinger Fellowship at Ohio University to aid in this work. 


\section{REFERENCES}

Ansorge, M. S., Zhou, M., Lira, A., Hen, R., Gingrich, J. A., 2004. Early-life blockade of the 5-HT transporter alters emotional behavior in adult mice. Science 306, 879-881.

Avitsur, R., 2017. Prenatal fluoxetine modifies the behavioral and hormonal responses to stress in male mice: role for glucocorticoid insensitivity. Behav Pharmacol 28, 345-355.

Avitsur, R., Grinshpahet, R., Goren, N., Weinstein, I., Kirshenboim, O., Chlebowski, N., 2016. Prenatal SSRI alters the hormonal and behavioral responses to stress in female mice: Possible role for glucocorticoid resistance. Horm Behav 84, 41-49.

Barker, J. M., Galea, L. A., 2008. Repeated estradiol administration alters different aspects of neurogenesis and cell death in the hippocampus of female, but not male, rats. Neuroscience 152,888 902.

Boulle, F., Pawluski, J. L., Homberg, J. R., Machiels, B., Kroeze, Y., Kumar, N., Steinbusch, H. W., Kenis, G., van den Hove, D. L., 2016a. Developmental fluoxetine exposure increases behavioral despair and alters epigenetic regulation of the hippocampal BDNF gene in adult female offspring. Hormones and Behavior 80, 47-57.

Boulle, F., Pawluski, J. L., Homberg, J. R., Machiels, B., Kroeze, Y., Kumar, N., Steinbusch, H. W., Kenis, G., Van den Hove, D. L., 2016b. Prenatal stress and early-life exposure to fluoxetine have enduring effects on anxiety and hippocampal BDNF gene expression in adult male offspring. Dev Psychobiol 58, 427-438.

Brown, H. K., Ray, J. G., Wilton, A. S., Lunsky, Y., Gomes, T., Vigod, S. N., 2017. Association Between Serotonergic Antidepressant Use During Pregnancy and Autism Spectrum Disorder in Children. Jama $317,1544-1552$.

Clements, C. C., Castro, V. M., Blumenthal, S. R., Rosenfield, H. R., Murphy, S. N., Fava, M., Erb, J. L., Churchill, S. E., Kaimal, A. J., Doyle, A. E., Robinson, E. B., Smoller, J. W., Kohane, I. S., Perlis, R. H., 2015. Prenatal antidepressant exposure is associated with risk for attention-deficit hyperactivity disorder but not autism spectrum disorder in a large health system. Molecular Psychiatry 20, 727-734.

Crawley, J. N., 2012. Translational animal models of autism and neurodevelopmental disorders. Dialogues Clin Neurosci 14, 293-305.

Dohler, K. D., Jarzab, B., Sickmoller, P. M., Kokocinska, D., Kaminski, M., Gubala, E., Achtelik, W., Wagiel, J., 1991. Influence of neurotransmitters on sexual differentiation of brain structure and function. Exp Clin Endocrinol 98, 99-109. 
El Marroun, H., White, T. J., van der Knaap, N. J., Homberg, J. R., Fernandez, G., Schoemaker, N. K., Jaddoe, V. W., Hofman, A., Verhulst, F. C., Hudziak, J. J., Stricker, B. H., Tiemeier, H., 2014. Prenatal exposure to selective serotonin reuptake inhibitors and social responsiveness symptoms of autism: population-based study of young children. Br J Psychiatry 205, 95-102.

Gemmel, M., Bogi, E., Ragan, C., Hazlett, M., Dubovicky, M., van den Hove, D. L., Oberlander, T. F., Charlier, T. D., Pawluski, J. L., 2018a. Perinatal selective serotonin reuptake inhibitor medication (SSRI) effects on social behaviors, neurodevelopment and the epigenome. Neurosci Biobehav Rev 85, 102-116.

Gemmel, M., Harmeyer, D., Bogi, E., Fillet, M., Hill, L. A., Hammond, G. L., Charlier, T. D., Pawluski, J. L., 2018b. Perinatal fluoxetine increases hippocampal neurogenesis and reverses the lasting effects of pregestational stress on serum corticosterone, but not on maternal behavior, in the rat dam. Behav Brain Res 339, 222-231.

Gemmel, M., Hazlett, M., Bogi, E., De Lacalle, S., Hill, L. A., Kokras, N., Hammond, G. L., Dalla, C., Charlier, T. D., Pawluski, J. L., 2017. Perinatal fluoxetine effects on social play, the HPA system, and hippocampal plasticity in pre-adolescent male and female rats: Interactions with pre-gestational maternal stress. Psychoneuroendocrinology 84, 159-171.

Gemmel, M., Kokras, N., Dalla, C., Pawluski, J. L., 2018c. Perinatal fluoxetine prevents the effect of pregestational maternal stress on $5-\mathrm{HT}$ in the PFC, but maternal stress has enduring effects on mPFC synaptic structure in offspring. Neuropharmacology 128, 168-180.

Gemmel, M., Rayen, I., Lotus, T., van Donkelaar, E., Steinbusch, H. W., De Lacalle, S., Kokras, N., Dalla, C., Pawluski, J. L., 2015. Developmental fluoxetine and prenatal stress effects on serotonin, dopamine, and synaptophysin density in the PFC and hippocampus of offspring at weaning. Developmental Psychobiology 232, 1231-1244.

Gemmel, M., Rayen, I., Lotus, T., van Donkelaar, E., Steinbusch, H. W., De Lacalle, S., Kokras, N., Dalla, C., Pawluski, J. L., 2016a. Developmental fluoxetine and prenatal stress effects on serotonin, dopamine, and synaptophysin density in the PFC and hippocampus of offspring at weaning. Dev Psychobiol 58, 315-327.

Gemmel, M., Rayen, I., van Donkelaar, E., Loftus, T., Steinbusch, H. W., Kokras, N., Dalla, C., Pawluski, J. L., 2016b. Gestational stress and fluoxetine treatment differentially affect plasticity, methylation and serotonin levels in the PFC and hippocampus of rat dams. Neuroscience 327, 32-43.

Gobinath, A. R., Workman, J. L., Chow, C., Lieblich, S. E., Galea, L. A., 2016. Maternal postpartum corticosterone and fluoxetine differentially affect adult male and female offspring on anxiety-like behavior, stress reactivity, and hippocampal neurogenesis. Neuropharmacology 101, 165-178. 
Hammels, C., Prickaerts, J., Kenis, G., Vanmierlo, T., Fischer, M., Steinbusch, H. W., van Os, J., van den Hove, D. L., Rutten, B. P., 2015. Differential susceptibility to chronic social defeat stress relates to the number of Dnmt3a-immunoreactive neurons in the hippocampal dentate gyrus. Psychoneuroendocrinology 51, 547-556.

Hammond, G. L., Lahteenmaki, P. L., 1983. A versatile method for the determination of serum cortisol binding globulin and sex hormone binding globulin binding capacities. Clin Chim Acta 132, 101-110.

Harrington, R. A., Lee, L. C., Crum, R. M., Zimmerman, A. W., Hertz-Picciotto, I., 2014. Prenatal SSRI use and offspring with autism spectrum disorder or developmental delay. Pediatrics 133, e1241-1248.

Hitti, F. L., Siegelbaum, S. A., 2014. The hippocampal CA2 region is essential for social memory. Nature 508, 88-92.

Huang, Y., Chen, S., Xu, H., Yu, X., Lai, H., Ho, G., Huang, Q., Shi, X., 2013. Pre-gestational stress alters stress-response of pubertal offspring rat in sexually dimorphic and hemispherically asymmetric manner. BMC Neurosci 14, 67.

Huang, Y., Shi, X., Xu, H., Yang, H., Chen, T., Chen, S., Chen, X., 2010. Chronic unpredictable stress before pregnancy reduce the expression of brain-derived neurotrophic factor and $\mathrm{N}$-methyl-D-aspartate receptor in hippocampus of offspring rats associated with impairment of memory. Neurochem Res 35 , 1038-1049.

Huang, Y., Xu, H., Li, H., Yang, H., Chen, Y., Shi, X., 2012. Pre-gestational stress reduces the ratio of 5HIAA to 5-HT and the expression of 5-HT1A receptor and serotonin transporter in the brain of foetal rat. BMC Neurosci 13, 22.

Ishiwata, H., Shiga, T., Okado, N., 2005. Selective serotonin reuptake inhibitor treatment of early postnatal mice reverses their prenatal stress-induced brain dysfunction. Neuroscience 133, 893-901.

Kepser, L. J., Homberg, J. R., 2015. The neurodevelopmental effects of serotonin: A behavioural perspective. Behav Brain Res 277C, 3-13.

Khatri, N., Simpson, K. L., Lin, R. C., Paul, I. A., 2014. Lasting neurobehavioral abnormalities in rats after neonatal activation of serotonin $1 \mathrm{~A}$ and $1 \mathrm{~B}$ receptors: possible mechanisms for serotonin dysfunction in autistic spectrum disorders. Psychopharmacology (Berl) 231, 1191-1200.

Kiryanova, V., Dyck, R. H., 2014. Increased aggression, improved spatial memory, and reduced anxietylike behaviour in adult male mice exposed to fluoxetine early in life. Developmental Neuroscience 36, 396-408. 
Kiser, D., Steemers, B., Branchi, I., Homberg, J. R., 2012. The reciprocal interaction between serotonin and social behaviour. Neurosci Biobehav Rev 36, 786-798.

Laplante, P., Diorio, J., Meaney, M. J., 2002. Serotonin regulates hippocampal glucocorticoid receptor expression via a 5-HT7 receptor. Brain Res Dev Brain Res 139, 199-203.

Loi, M., Koricka, S., Lucassen, P. J., Joels, M., 2014. Age- and Sex-Dependent Effects of Early Life Stress on Hippocampal Neurogenesis. Front Endocrinol (Lausanne) 5, 13.

Malm, H., Brown, A. S., Gissler, M., Gyllenberg, D., Hinkka-Yli-Salomaki, S., McKeague, I. W., Weissman, M., Wickramaratne, P., Artama, M., Gingrich, J. A., Sourander, A., 2016. Gestational Exposure to Selective Serotonin Reuptake Inhibitors and Offspring Psychiatric Disorders: A National Register-Based Study. J Am Acad Child Adolesc Psychiatry 55, 359-366.

Man, K. K., Tong, H. H., Wong, L. Y., Chan, E. W., Simonoff, E., Wong, I. C., 2015. Exposure to selective serotonin reuptake inhibitors during pregnancy and risk of autism spectrum disorder in children: a systematic review and meta-analysis of observational studies. Neurosci Biobehav Rev 49, 82-89.

Mezzacappa, A., Lasica, P. A., Gianfagna, F., Cazas, O., Hardy, P., Falissard, B., Sutter-Dallay, A. L., Gressier, F., 2017. Risk for Autism Spectrum Disorders According to Period of Prenatal Antidepressant Exposure: A Systematic Review and Meta-analysis. JAMA Pediatr 171, 555-563.

Oberlander, T. F., Gingrich, J. A., Ansorge, M. S., 2009. Sustained neurobehavioral effects of exposure to SSRI antidepressants during development: molecular to clinical evidence. Clin Pharmacol Ther 86, 672677.

Oberlander, T. F., Warburton, W., Misri, S., Aghajanian, J., Hertzman, C., 2006. Neonatal outcomes after prenatal exposure to selective serotonin reuptake inhibitor antidepressants and maternal depression using population-based linked health data. Arch Gen Psychiatry 63, 898-906.

Oberlander, T. F., Zwaigenbaum, L., 2017. Disentangling Maternal Depression and Antidepressant Use During Pregnancy as Risks for Autism in Children. Jama 317, 1533-1534.

Olivier, J. D., Akerud, H., Kaihola, H., Pawluski, J. L., Skalkidou, A., Hogberg, U., Sundstrom-Poromaa, I., 2013. The effects of maternal depression and maternal selective serotonin reuptake inhibitor exposure on offspring. Front Cell Neurosci 7, 73.

Park, M., Oberlander, T. F., 2018. In-utero Selective Serotonin Reuptake Inhibitor Antidepressant Exposure: Fetal Programing and Developmental Interactions With Context. Clin Pharmacol Ther. 
Pawluski, J. L., Rayen, I., Niessen, N. A., Kristensen, S., Donkelaar, E. L. v., Balthazart, J., Steinbusch, H. W., Charlier, T. D., 2012a. Developmental fluoxetine exposure differentially alters central and peripheral measures of the HPA system in adolescent male and female offspring. Neuroscience 220, 131-141.

Pawluski, J. L., Rayen, I., Niessen, N. A., Kristensen, S., van Donkelaar, E. L., Balthazart, J., Steinbusch, H. W., Charlier, T. D., 2012b. Developmental fluoxetine exposure differentially alters central and peripheral measures of the HPA system in adolescent male and female offspring. Neuroscience 220, 131-141.

Rayen, I., Gemmel, M., Pauley, G., Steinbusch, H. W., Pawluski, J. L., 2015. Developmental exposure to SSRIs, in addition to maternal stress, has long-term sex-dependent effects on hippocampal plasticity. Psychopharmacology (Berl) 232, 1231-1244.

Rayen, I., Steinbusch, H. W., Charlier, T. D., Pawluski, J. L., 2013. Developmental fluoxetine exposure and prenatal stress alter sexual differentiation of the brain and reproductive behavior in male rat offspring. Psychoneuroendocrinology 38, 1618-1629.

Rayen, I., Steinbusch, H. W., Charlier, T. D., Pawluski, J. L., 2014. Developmental fluoxetine exposure facilitates sexual behavior in female offspring. Psychopharmacology (Berl) 231, 123-133.

Rodriguez-Porcel, F., Green, D., Khatri, N., Harris, S. S., May, W. L., Lin, R. C., Paul, I. A., 2011. Neonatal exposure of rats to antidepressants affects behavioral reactions to novelty and social interactions in a manner analogous to autistic spectrum disorders. Anat Rec (Hoboken) 294, 1726-1735.

Simpson, K. L., Weaver, K. J., de Villers-Sidani, E., Lu, J. Y., Cai, Z., Pang, Y., Rodriguez-Porcel, F., Paul, I. A., Merzenich, M., Lin, R. C., 2011. Perinatal antidepressant exposure alters cortical network function in rodents. Proc Natl Acad Sci U S A 108, 18465-18470.

Stevenson, E. L., Caldwell, H. K., 2014. Lesions to the CA2 region of the hippocampus impair social memory in mice. Eur J Neurosci 40, 3294-3301.

Stewart, D. E., 2011. Clinical practice. Depression during pregnancy. N Engl J Med 365, 1605-1611.

Svirsky, N., Levy, S., Avitsur, R., 2016. Prenatal exposure to selective serotonin reuptake inhibitors (SSRI) increases aggression and modulates maternal behavior in offspring mice. Developmental Psychobiology $58,71-82$.

Taylor, S. E., Klein, L. C., Lewis, B. P., Gruenewald, T. L., Gurung, R. A., Updegraff, J. A., 2000. Biobehavioral responses to stress in females: tend-and-befriend, not fight-or-flight. Psychol Rev 107, 411-429. 
van Kerkhof, L. W., Damsteegt, R., Trezza, V., Voorn, P., Vanderschuren, L. J., 2013. Social play behavior in adolescent rats is mediated by functional activity in medial prefrontal cortex and striatum. Neuropsychopharmacology 38, 1899-1909.

Veenema, A. H., 2012. Toward understanding how early-life social experiences alter oxytocin- and vasopressin-regulated social behaviors. Horm Behav 61, 304-312.

Veenema, A. H., Bredewold, R., De Vries, G. J., 2012. Vasopressin regulates social recognition in juvenile and adult rats of both sexes, but in sex- and age-specific ways. Horm Behav 61, 50-56.

Walker, S. E., Papilloud, A., Huzard, D., Sandi, C., 2016. The link between aberrant hypothalamicpituitary-adrenal axis activity during development and the emergence of aggression-Animal studies. Neuroscience \& Biobehavioral Reviews. 
Table 1. Chronic unpredictable stress (CUS) schedule prior to gestation. Females in the pre-gestational stress group were housed individually on day 0 and subjected to chronic unpredictable stress consisting of 0-2 stressors per day for 3 weeks. Previously published in Gemmel et al, 2017c.

\begin{tabular}{|l|l|l|l|l|}
\hline Day of CUS & \multicolumn{1}{|c|}{ Stressor 1 } & Duration of Stressor 1 & \multicolumn{1}{c|}{ Stressor 2 } & Duration of stressor 2 \\
\hline $\mathbf{1}$ & Damp Bedding & 8h day time & Overcrowding & 24 hours \\
\hline $\mathbf{2}$ & Restraint Stress & 1 hour & Cage Rotation & 12 hours \\
\hline $\mathbf{3}$ & Overcrowding & 24 hours & - & - \\
\hline $\mathbf{4}$ & Food Depravation & $8-12$ hours & Swim Stress & 5 minutes \\
\hline $\mathbf{5}$ & Restraint Stress & 1 hour & Food Depravation & 12 hours \\
\hline $\mathbf{6}$ & Swim Stress & 5 minutes & Overcrowding & 24 hours \\
\hline $\mathbf{7}$ & Restraint Stress & 1 hour & - & - \\
\hline $\mathbf{8}$ & Damp Bedding & 8 hours & Cage Rotation & 12 hours \\
\hline $\mathbf{9}$ & Restraint Stress & 1 hour & Food Depravation & 12 hours \\
\hline $\mathbf{1 0}$ & Damp Bedding & 8 hours & Overcrowding & 24 hours \\
\hline $\mathbf{1 1}$ & - & - & - & - \\
\hline $\mathbf{1 2}$ & Cage Rotation & 12 hours & Swim Stress & 5 minutes \\
\hline $\mathbf{1 3}$ & Restraint Stress & 1 hour & Swim Stress & 5 minutes \\
\hline $\mathbf{1 4}$ & Damp Bedding & 8 hours & Overcrowding & 24 hours \\
\hline $\mathbf{1 5}$ & Restraint Stress & 1 hour & Cage Rotation & 12 hours \\
\hline $\mathbf{1 6}$ & Food Depravation & 12 hours & Overcrowding & 24 hours \\
\hline $\mathbf{1 7}$ & Restraint Stress & 1 hour & Restraint Stress & 1 hour \\
\hline $\mathbf{1 8}$ & Overcrowding & 24 hours & - & - \\
\hline $\mathbf{1 9}$ & Swim Stress & 5 minutes & Swim Stress & 5 minutes \\
\hline $\mathbf{2 0}$ & Cage Rotation & 1 hour & Damp Bedding & 8 hours \\
\hline $\mathbf{2 1}$ & Overcrowding & 24 hours & Food Depravation & 12 hours \\
\hline
\end{tabular}


Table 2. Significant three-way ANOVA main effects with Condition (pre-gestational stress/control), Treatment (fluoxetine/vehicle) and Sex (female/male) on measures of social behaviors, HPA axis, and synaptic outcomes.

\begin{tabular}{|c|c|c|c|}
\hline & Main Effect of Condition & Main Effect of Treatment & Main Effect of Sex \\
\hline \multicolumn{4}{|c|}{ Social Behavior } \\
\hline & $\begin{array}{l}\text { sniffing }(\mathrm{F}(1,79)=15.2, \mathrm{p}=0.0002) \\
\text { crawling }(\mathrm{F}(1,79)=3.97, \mathrm{p}=0.049) \\
\text { social investigation }(\mathrm{F}(1,79)=15.67, \mathrm{p}=0.0002) \\
\text { all scored behavior }(\mathrm{F}(1,79)=5.89, \mathrm{p}=0.02)\end{array}$ & $\begin{array}{l}\text { social investigation }(\mathrm{F}(1,79)=6.64, \mathrm{p}=0.01) \\
\text { all scored behavior }(\mathrm{F}(1,79)=4.57, \mathrm{p}=0.04)\end{array}$ & $\begin{array}{l}\text { sniffing }(F(1,79)=7.10, p=0.009) \\
\text { social investigation }(F(1,79)=5.16, p=0.03)\end{array}$ \\
\hline \multicolumn{4}{|c|}{ ( } \\
\hline & $\begin{array}{l}\text { Corticosterone }(\mathrm{F}(1,74)=5.33, \mathrm{p}=0.02) \\
\text { CBG }(\mathrm{F}(1,79)=6.97, \mathrm{p}=0.01)\end{array}$ & & $\begin{array}{l}\text { free corticosterone }(\mathrm{F}(1,74)=68.26, \mathrm{p}<0.00001) \\
\text { Corticosterone }(\mathrm{F}(1,74)=187.52, \mathrm{p}<0.00001) \\
\text { CBG }(\mathrm{F}(1,79)=678.48, \mathrm{p}<0.00001) \\
\text { GR density in DG }(\mathrm{F}(1,40)=12.84, \mathrm{p}=0.0009) \\
\text { GR density in CA3 }(\mathrm{F}(1,40)=10.26, \mathrm{p}=0.003)\end{array}$ \\
\hline \multirow{2}{*}{ Hippocampus } & & 8 & \\
\hline & DCX-ir cells $(F(1,40)=8.54, p=0.006)$ & PSD-95-ir in CA2 $(\mathrm{F}(1,40)=11.57, \mathrm{p}=0.002)$ & \\
\hline
\end{tabular}


Table 3. Mean $( \pm$ SEM) time to first interaction and duration ( $s$ ) in scored behaviors with a novel stimulus conspecific. *denotes significance, ^denotes significant main effect of treatment $(\mathrm{p}<0.05)$. $(\mathrm{n}=8$ $16 /$ sex/group)

\begin{tabular}{l|cccc}
\hline & CV & CF & PGSV & PGSF \\
\hline & & \multicolumn{3}{c}{ Female } \\
\hline Time to first interaction & $13.6 \pm 2.6$ & $12.4 \pm 2.1^{\wedge}$ & $19.8 \pm 3.5$ & $6.1 \pm 1.6^{\wedge}$ \\
Sniffing & $86.5 \pm 6.5$ & $114.3 \pm 7.8^{\wedge}$ & $70.0 \pm 8.6^{*}$ & $94.4 \pm 8.1^{\wedge *}$ \\
Following & $32.5 \pm 3.4$ & $48.2 \pm 8.2^{\wedge}$ & $26.3 \pm 6.0$ & $50.6 \pm 4.7^{\wedge}$ \\
Crawling & $55.0 \pm 6.8$ & $48.2 \pm 6.8$ & $58.8 \pm 7.3$ & $59.4 \pm 6.4$ \\
Grooming partner & $6.0 \pm 1.8$ & $7.9 \pm 1.5^{\wedge}$ & $2.5 \pm 0.9$ & $8.3 \pm 2.5^{\wedge}$ \\
Running Away & $10.0 \pm 2.8$ & $14.3 \pm 2.7$ & $18.1 \pm 4.4$ & $11.1 \pm 1.4$ \\
Self-grooming & $16.8 \pm 4.9$ & $8.9 \pm 1.4$ & $15.9 \pm 3.0$ & $14.9 \pm 4.1$ \\
\hline & & & Male & \\
\hline Time to first interaction & $9.2 \pm 1.7$ & $9.5 \pm 1.3$ & $10.4 \pm 3.1$ & $18.2 \pm 4.8$ \\
Sniffing & $123.8 \pm 6.7$ & $115.9 \pm 7.2$ & $93.1 \pm 8.1^{*}$ & $93.5 \pm 8.9^{*}$ \\
Following & $50.4 \pm 4.9$ & $57.8 \pm 4.9$ & $35.0 \pm 4.8^{*}$ & $31.0 \pm 5.8^{*}$ \\
Crawling & $45.4 \pm 5.1$ & $42.8 \pm 4.4$ & $59.4 \pm 6.4$ & $50.0 \pm 7.0$ \\
Grooming partner & $10.8 \pm 1.9$ & $9.7 \pm 2.0$ & $6.3 \pm 2.3$ & $7.0 \pm 1.7^{*}$ \\
Running Away & $7.1 \pm 2.2$ & $11.9 \pm 1.9^{\wedge}$ & $5.0 \pm 1.3$ & $16.0 \pm 3.5^{\wedge}$ \\
Self-grooming & $9.1 \pm 2.3$ & $13.2 \pm 2.3^{\wedge}$ & $8.5 \pm 1.8$ & $15.8 \pm 2.5^{\wedge}$ \\
\hline
\end{tabular}


Table 4. Mean $( \pm$ SEM) serum corticosterone $(n M), C B G(n M)$ and free corticosterone ( $n=8-16 /$ group), and glucocorticoid receptor (GR) density (OD) in the hippocampus and PVN (n=5-6/group). * denotes significant main effect of pre-gestational stress $(\mathrm{p}<0.05) . \mathrm{CV}=$ control + vehicle, $\mathrm{CF}=$ control + fluoxetine, $\mathrm{PGSV}=$ pre-gestational stress + vehicle, $\mathrm{PGSF}=$ pre-gestational stress + fluoxetine.

\begin{tabular}{l|cccc}
\hline & CV & CF & PGSV & PGSF \\
& \multicolumn{4}{|c}{ Female } \\
\hline Corticosterone (nM) & $1974.7 \pm 348.47$ & $2099.4 \pm 192.9$ & $1726.0 \pm 188.4^{*}$ & $1383.6 \pm 153.1^{*}$ \\
CBG (nM) & $1980.1 \pm 127.3$ & $2023.4 \pm 58.4$ & $1654.3 \pm 84.5^{*}$ & $1931.4 \pm 70.7^{*}$ \\
Free corticosterone (nM) & $100.6 \pm 17.6$ & $101.9 \pm 8.9$ & $105.8 \pm 13.1$ & $73.8 \pm 9.7$ \\
GR-ir CA2 & $0.10 \pm 0.01$ & $0.10 \pm 0.02$ & $0.08 \pm 0.01$ & $0.09 \pm 0.01$ \\
GR-ir PVN & $0.23 \pm 0.03$ & $0.18 \pm 0.01$ & $0.17 \pm 0.01$ & $0.18 \pm 0.04$ \\
\hline & & & & \\
\hline Corticosterone (nM) & $226.6 \pm 41.0$ & $284.7 \pm 63.6$ & $279.4 \pm 51.4$ & $143.0 \pm 48.1$ \\
CBG (nM) & $765.0 \pm 19.2$ & $746.0 \pm 26.1$ & $733.7 \pm 29.8$ & $726.9 \pm 31.5$ \\
Free corticosterone (nM) & $29.7 \pm 5.4$ & $41.1 \pm 10.0$ & $39.8 \pm 8.3$ & $19.6 \pm 6.6$ \\
GR-ir CA2 & $0.07 \pm 0.004$ & $0.07 \pm 0.01$ & $0.08 \pm 0.01$ & $0.08 \pm 0.01$ \\
GR-ir PVN & $0.17 \pm 0.03$ & $0.21 \pm 0.02$ & $0.16 \pm 0.01$ & $0.18 \pm 0.01$ \\
\hline
\end{tabular}


Table 5. Mean $( \pm \mathrm{SEM})$ synaptic protein density (OD) in the hippocampus ( $\mathrm{n}=6 /$ group). $\mathrm{CV}=$ control + vehicle, $\mathrm{CF}=$ control + fluoxetine, $\mathrm{PGSV}=$ pre-gestational stress + vehicle, $\mathrm{PGSF}=$ pre-gestational stress + fluoxetine.

\begin{tabular}{c|cccc}
\hline & CV & CF & PGSV & PGSF \\
\hline Synaptophysin & \multicolumn{3}{|c}{ Female } \\
DG & $0.19 \pm 0.03$ & $0.16 \pm 0.02$ & $0.15 \pm 0.01$ & $0.20 \pm 0.03$ \\
CA2 & $0.05 \pm 0.02$ & $0.07 \pm 0.03$ & $0.02 \pm 0.01$ & $0.07 \pm 0.02$ \\
CA3 & $0.16 \pm 0.03$ & $0.13 \pm 0.01$ & $0.11 \pm 0.01$ & $0.15 \pm 0.02$ \\
PSD-95 & & & $0.04 \pm 0.006$ & $0.04 \pm 0.005$ \\
DG & $0.04 \pm 0.007$ & $0.05 \pm 0.004$ & $0.05 \pm 0.006$ & $0.04 \pm 0.004$ \\
CA3 & $0.05 \pm 0.007$ & $0.04 \pm 0.005$ & & \\
\hline Synaptophysin & & & Male & \\
DG & $0.22 \pm 0.02$ & $0.18 \pm 0.02$ & $0.18 \pm 0.03$ & $0.21 \pm 0.03$ \\
CA2 & $0.07 \pm 0.01$ & $0.07 \pm 0.01$ & $0.07 \pm 0.02$ & $0.10 \pm 0.01$ \\
CA3 & $0.16 \pm 0.01$ & $0.13 \pm 0.01$ & $0.14 \pm 0.03$ & $0.15 \pm 0.02$ \\
PSD-95 & & & & \\
DG & $0.06 \pm 0.01$ & $0.04 \pm 0.004$ & $0.04 \pm 0.006$ & $0.03 \pm 0.004$ \\
CA3 & $0.05 \pm 0.009$ & $0.04 \pm 0.003$ & $0.05 \pm 0.006$ & $0.06 \pm 0.03$ \\
\hline
\end{tabular}


Figure 1
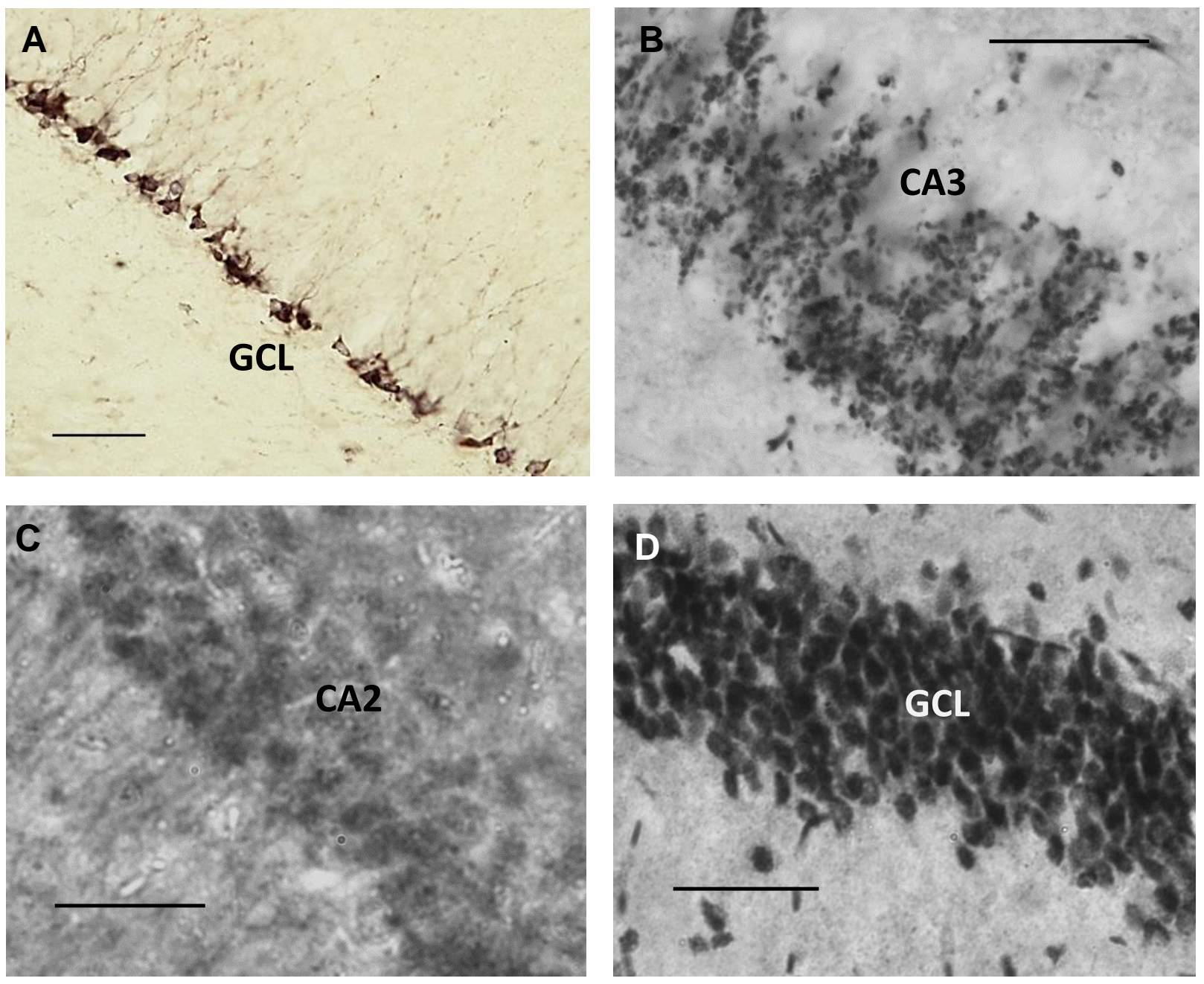
Figure 2

\section{Female}

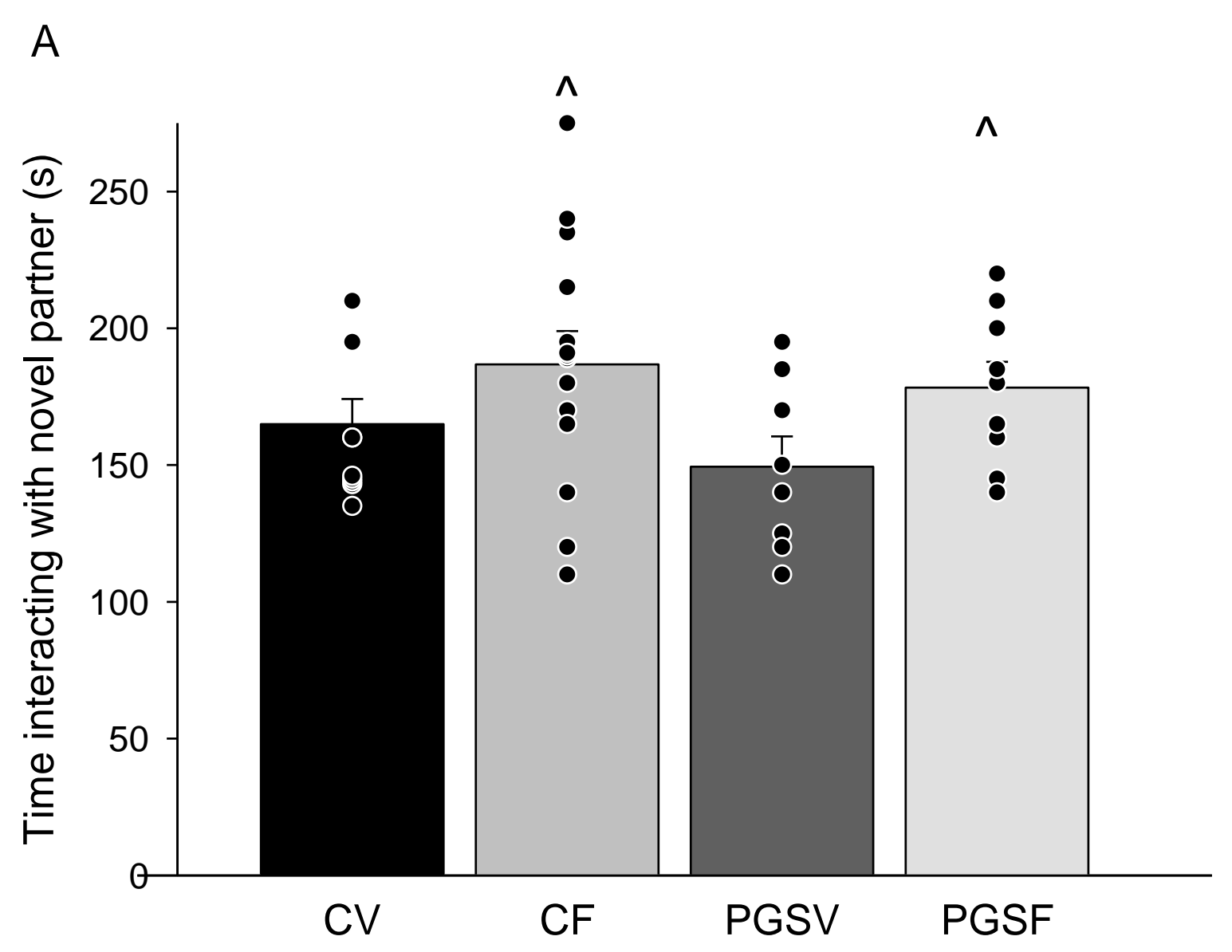

B

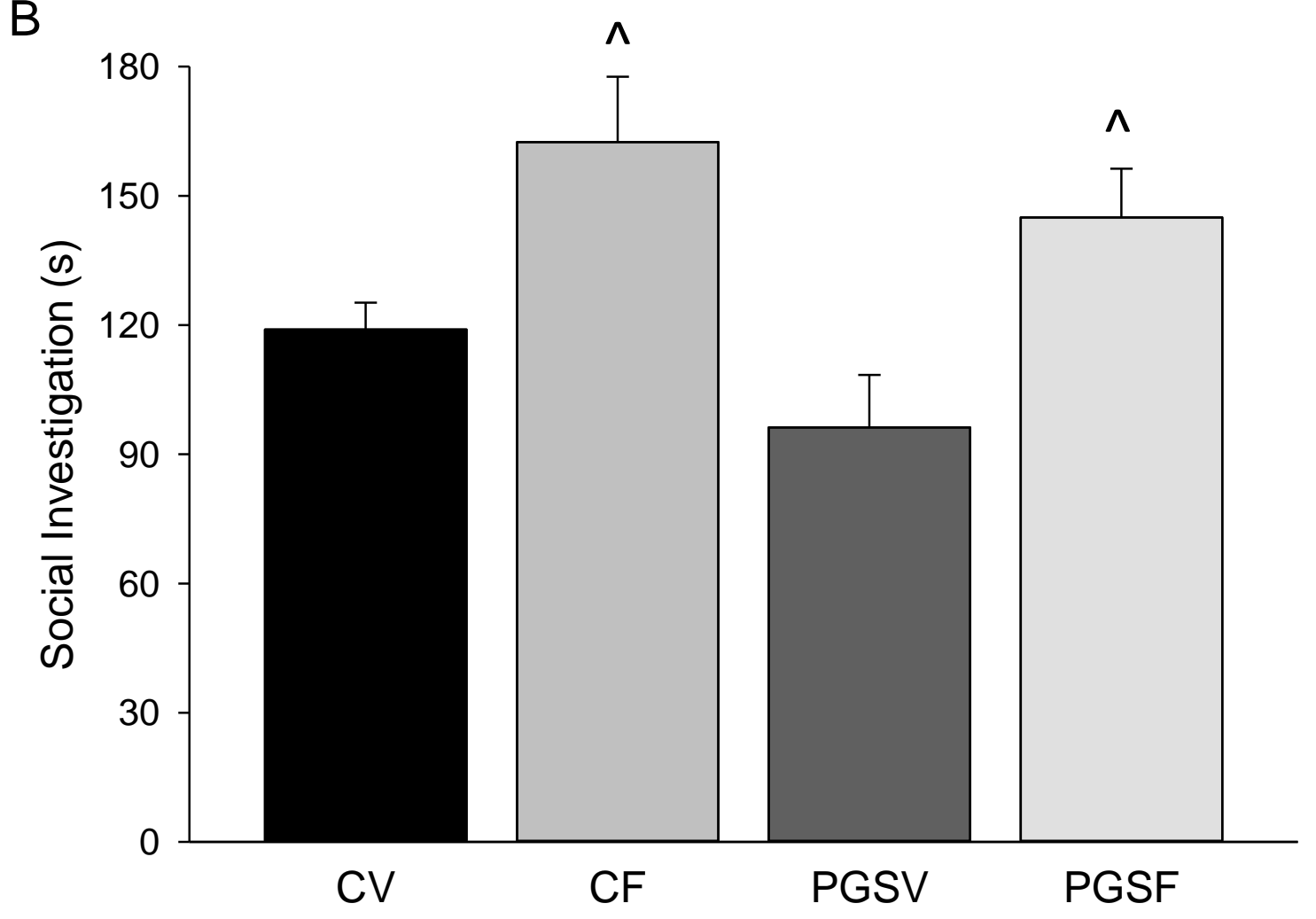

C

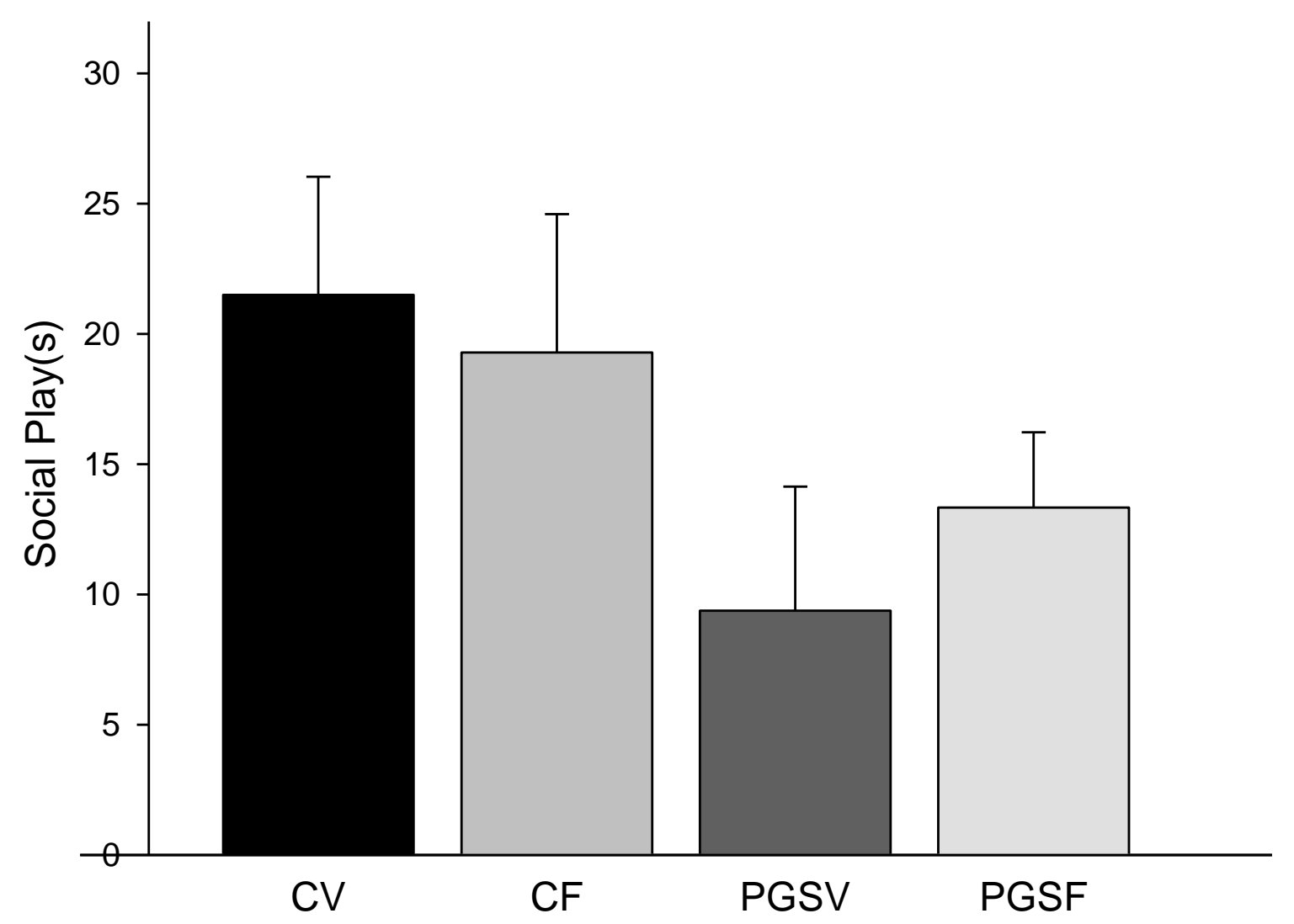

Male

D

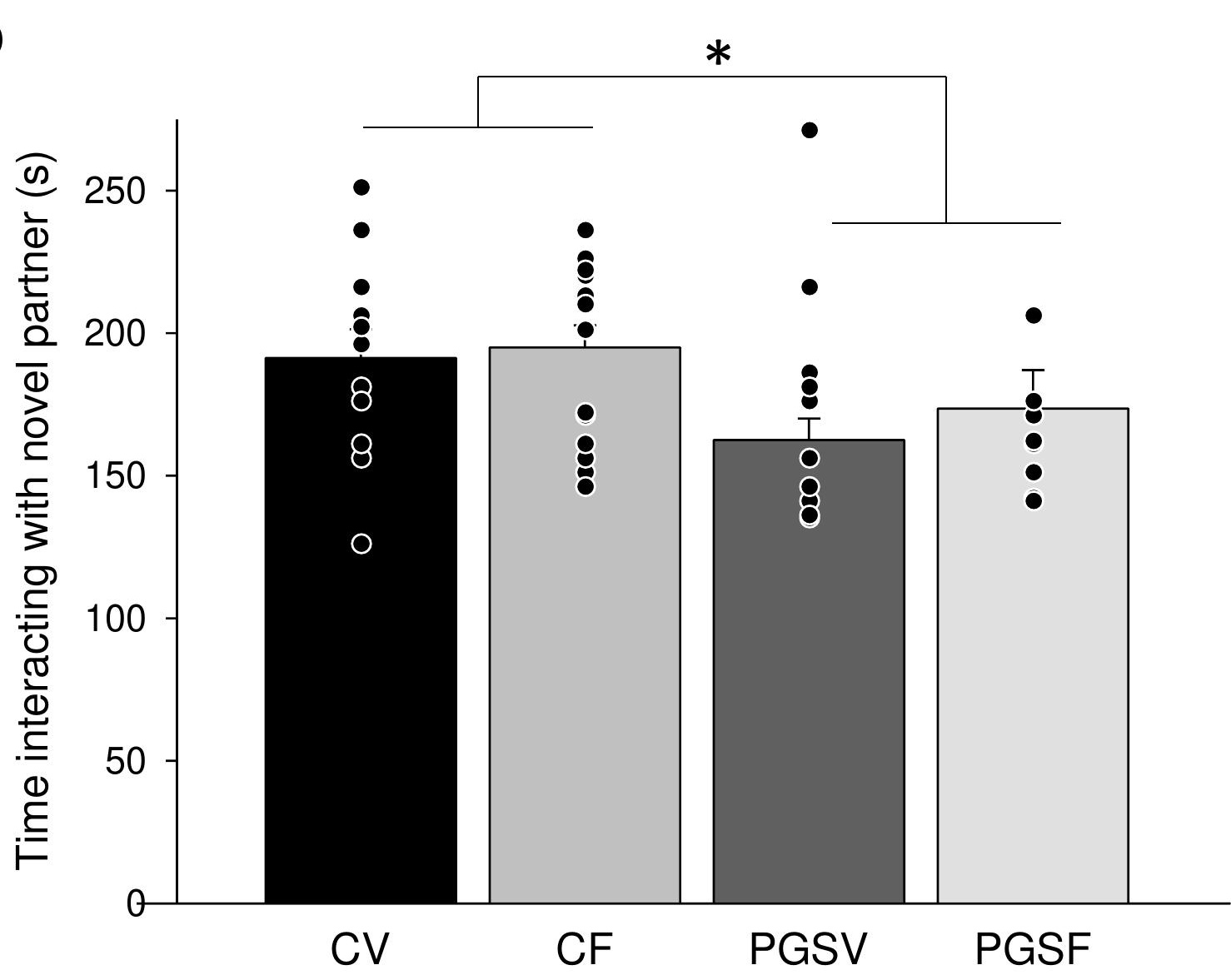

E

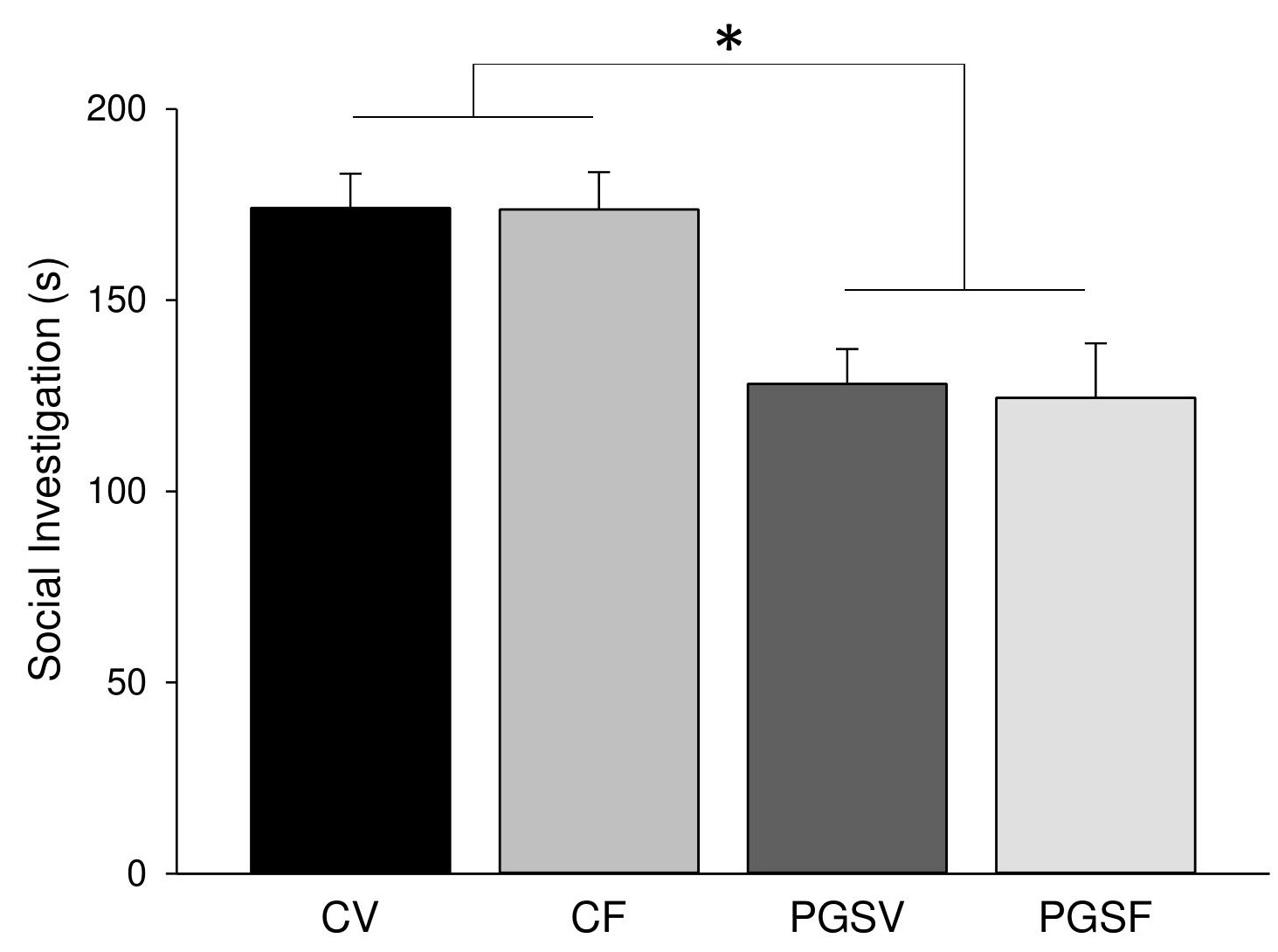

F

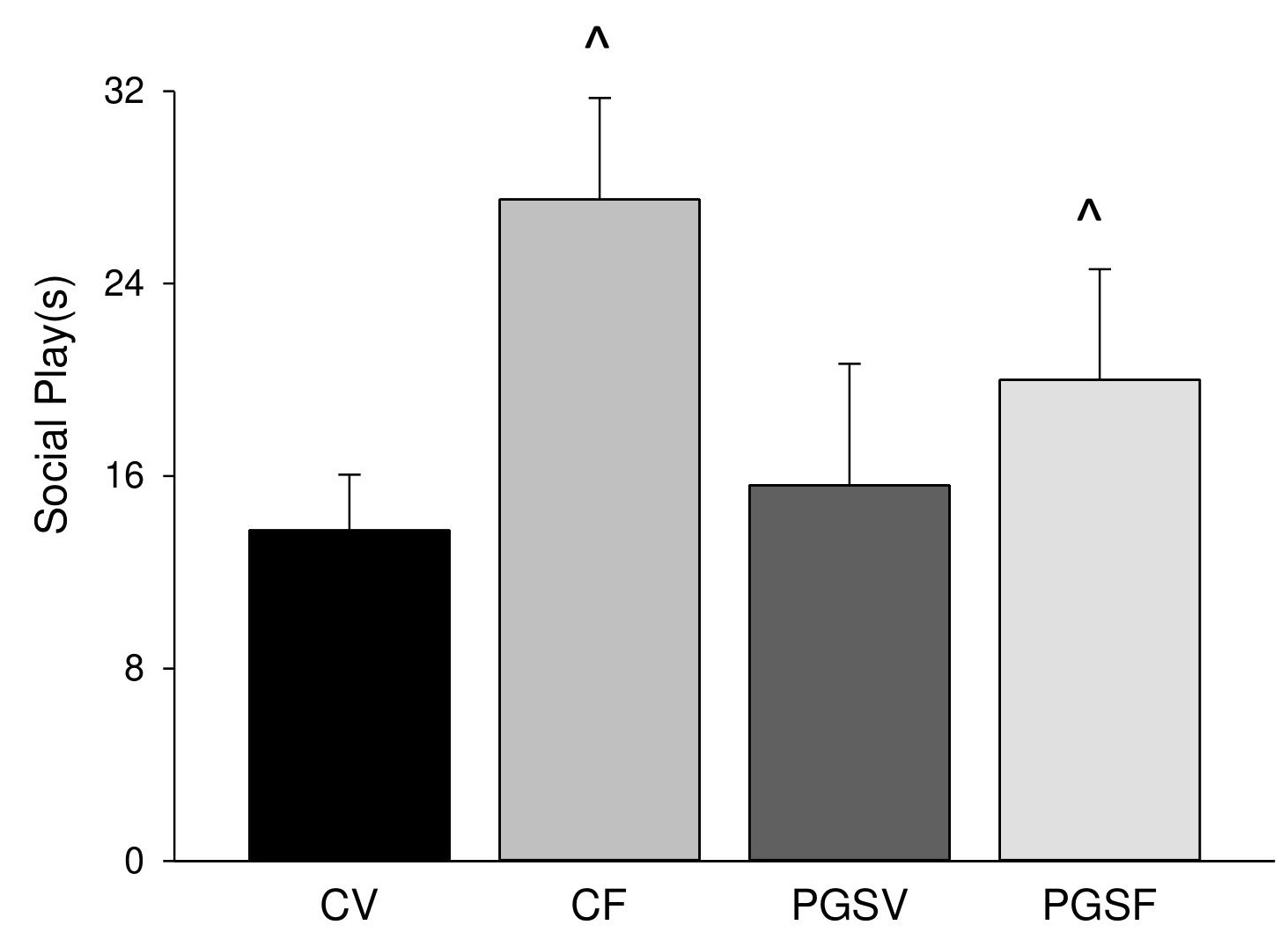


Figure 3

\section{Female}

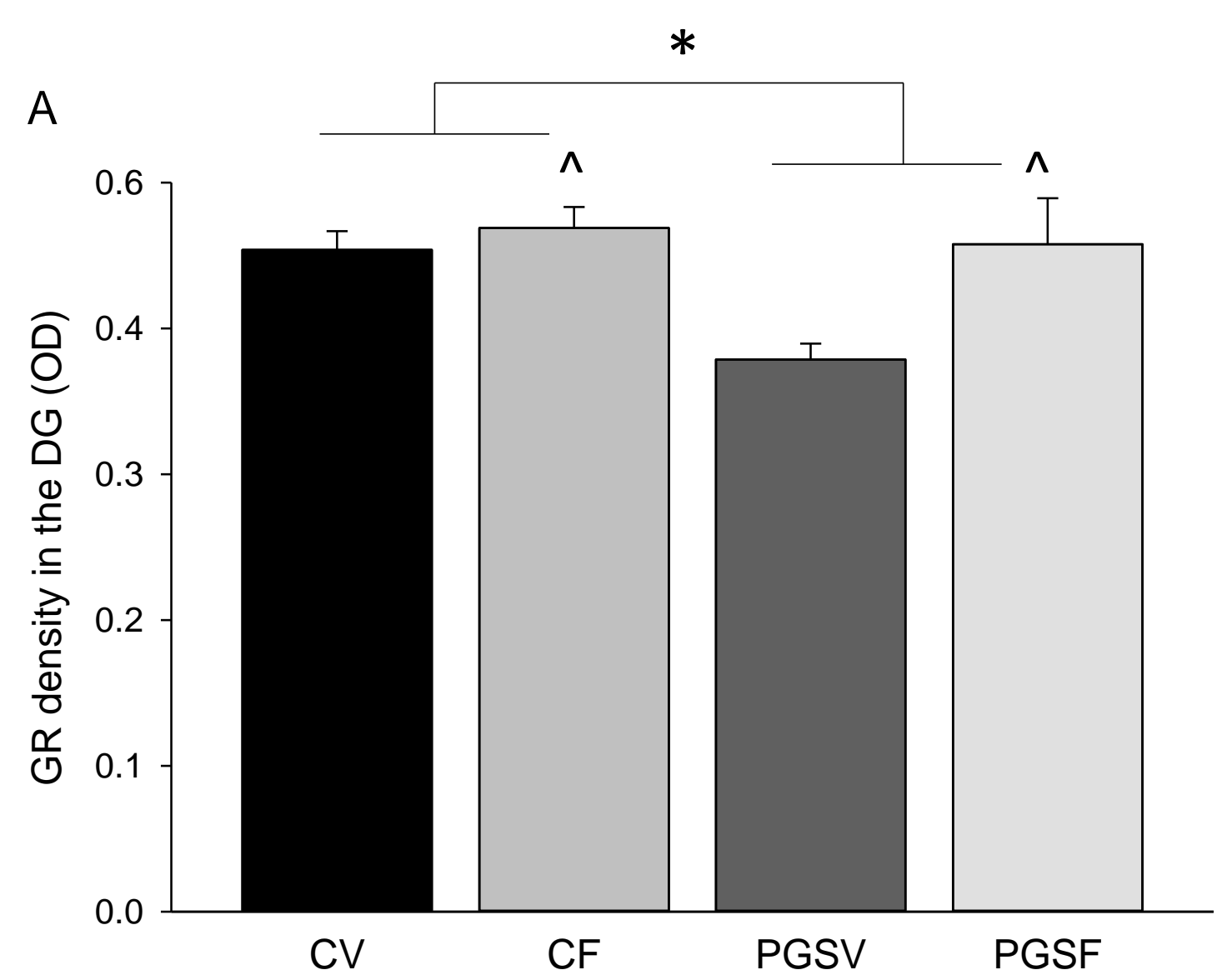

B

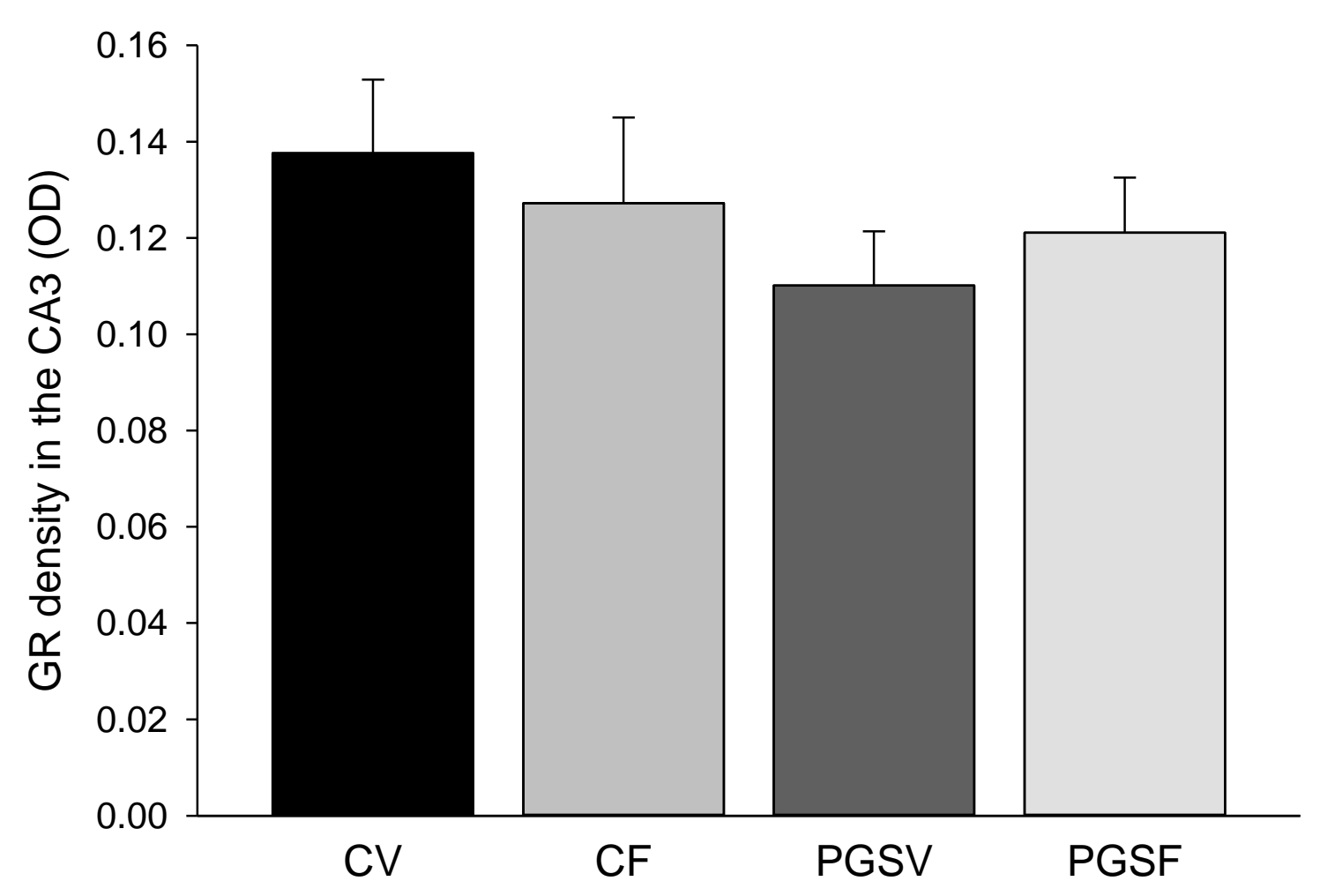

C

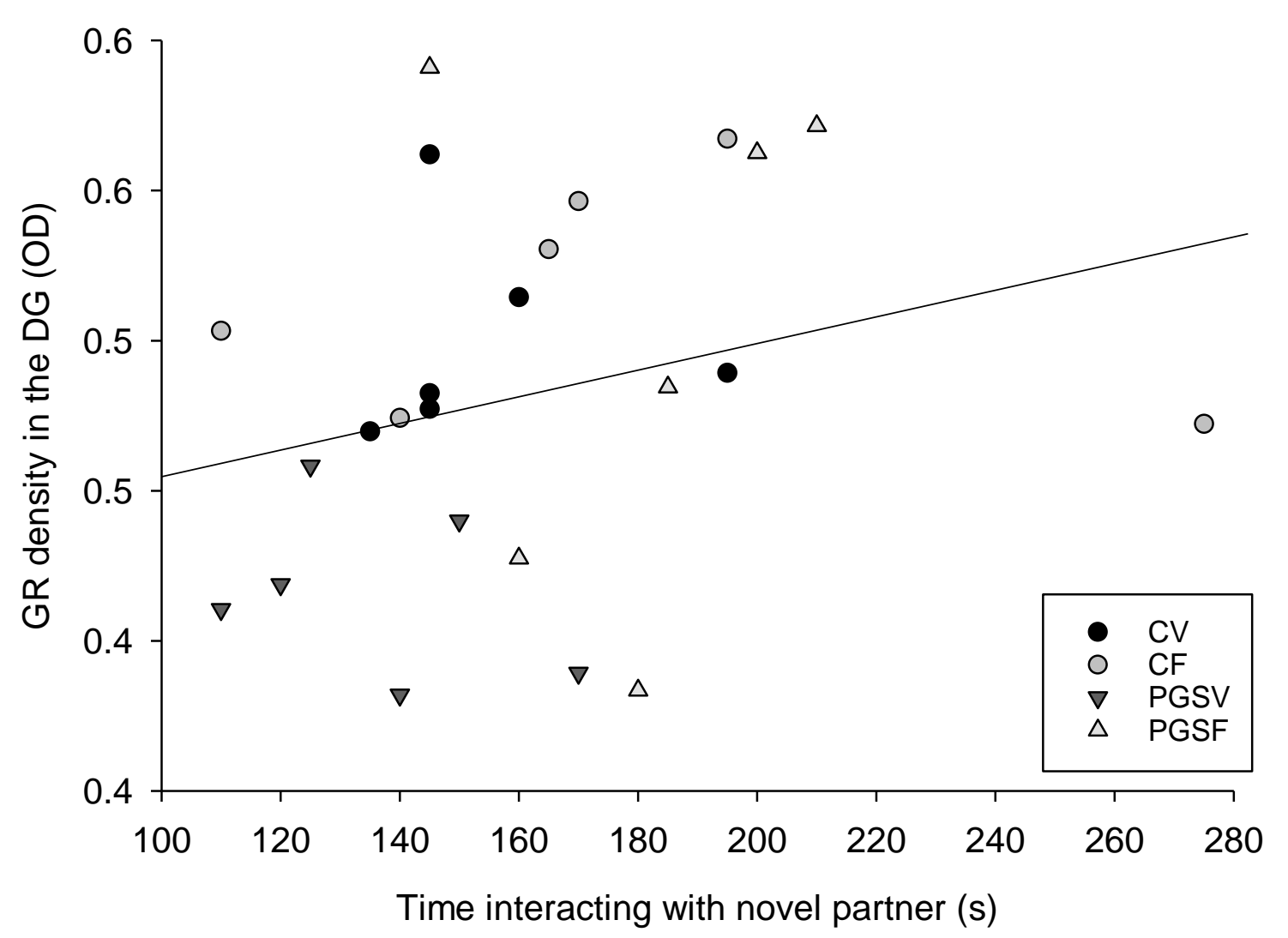

Male

D

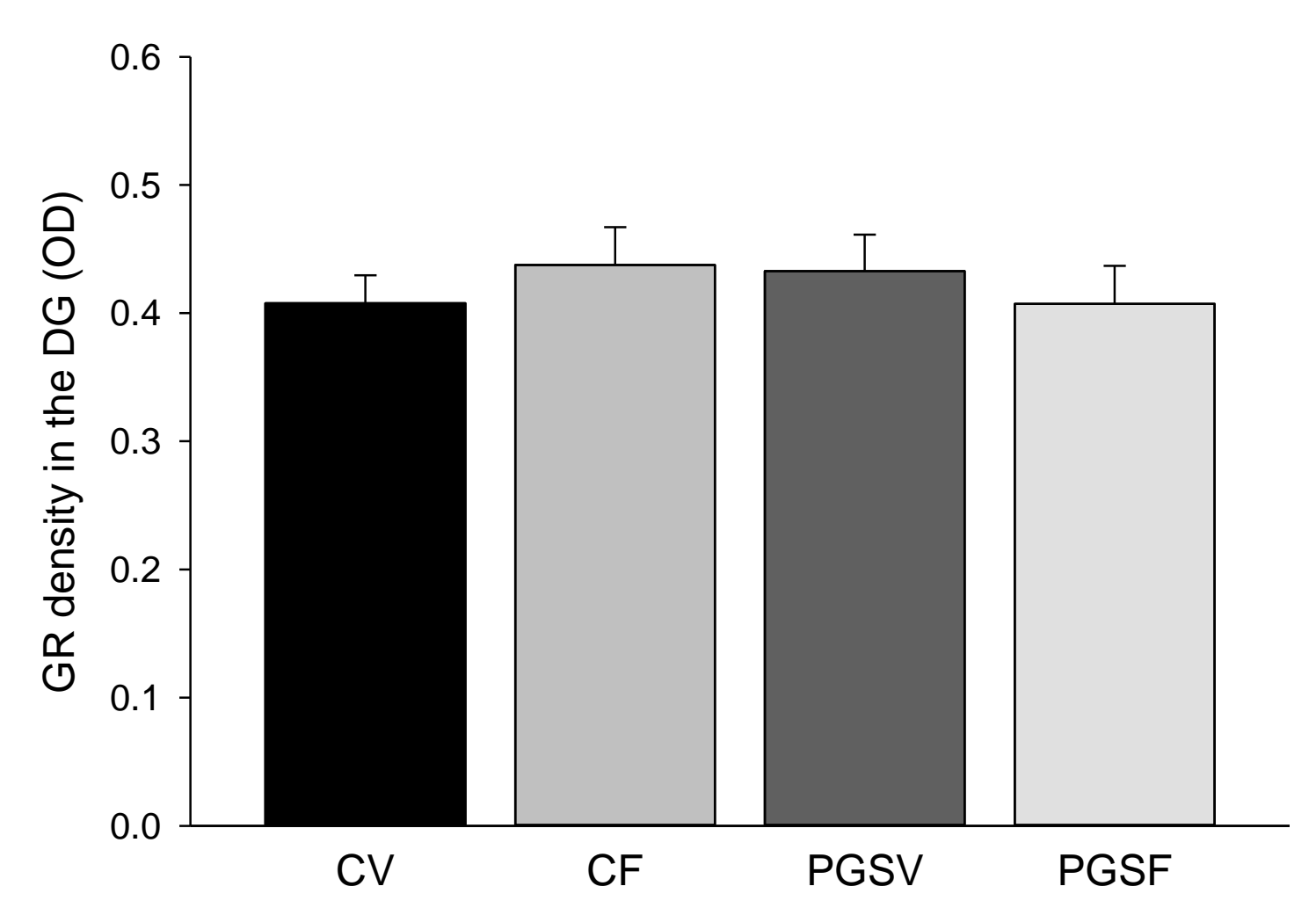

E

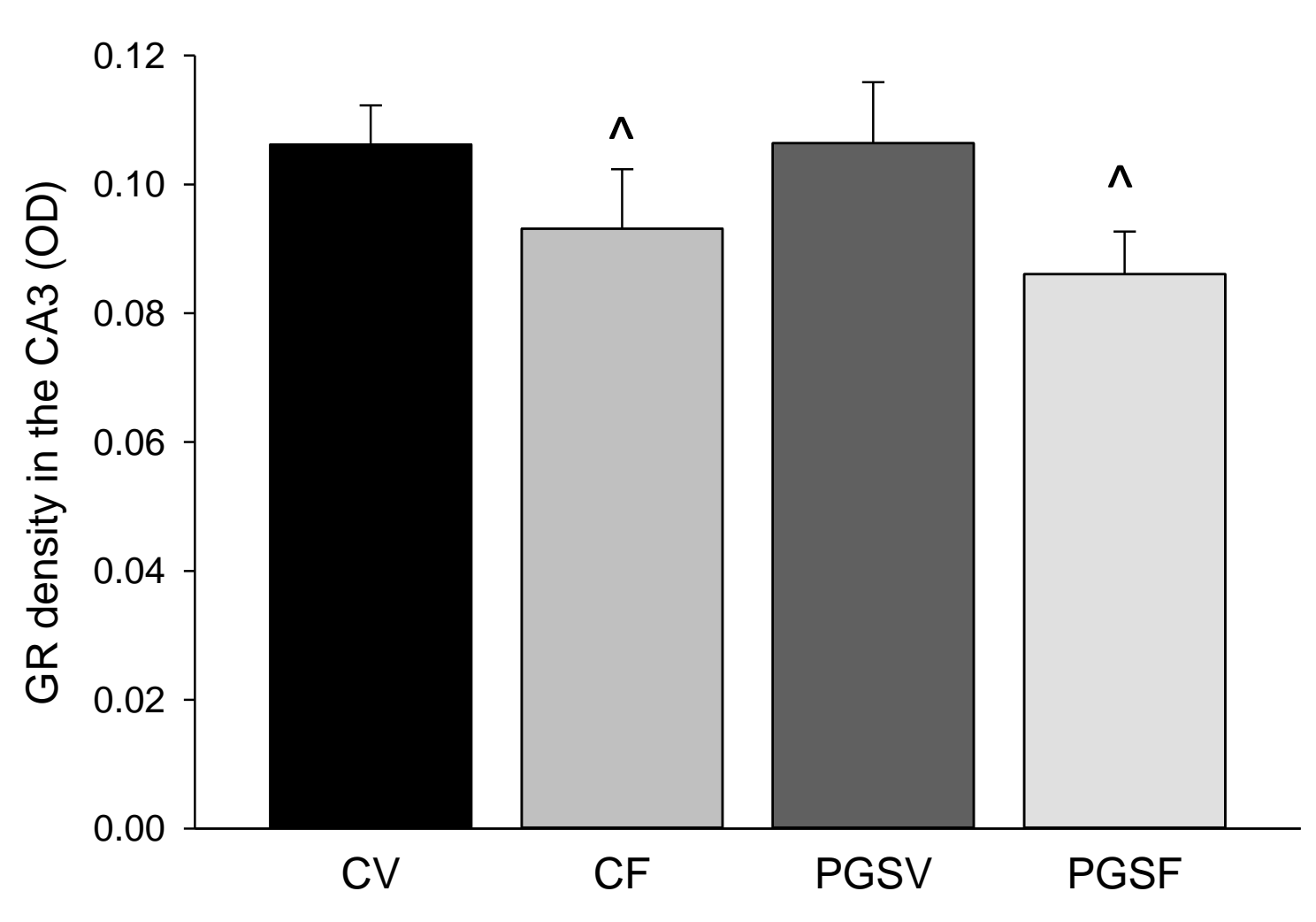

F

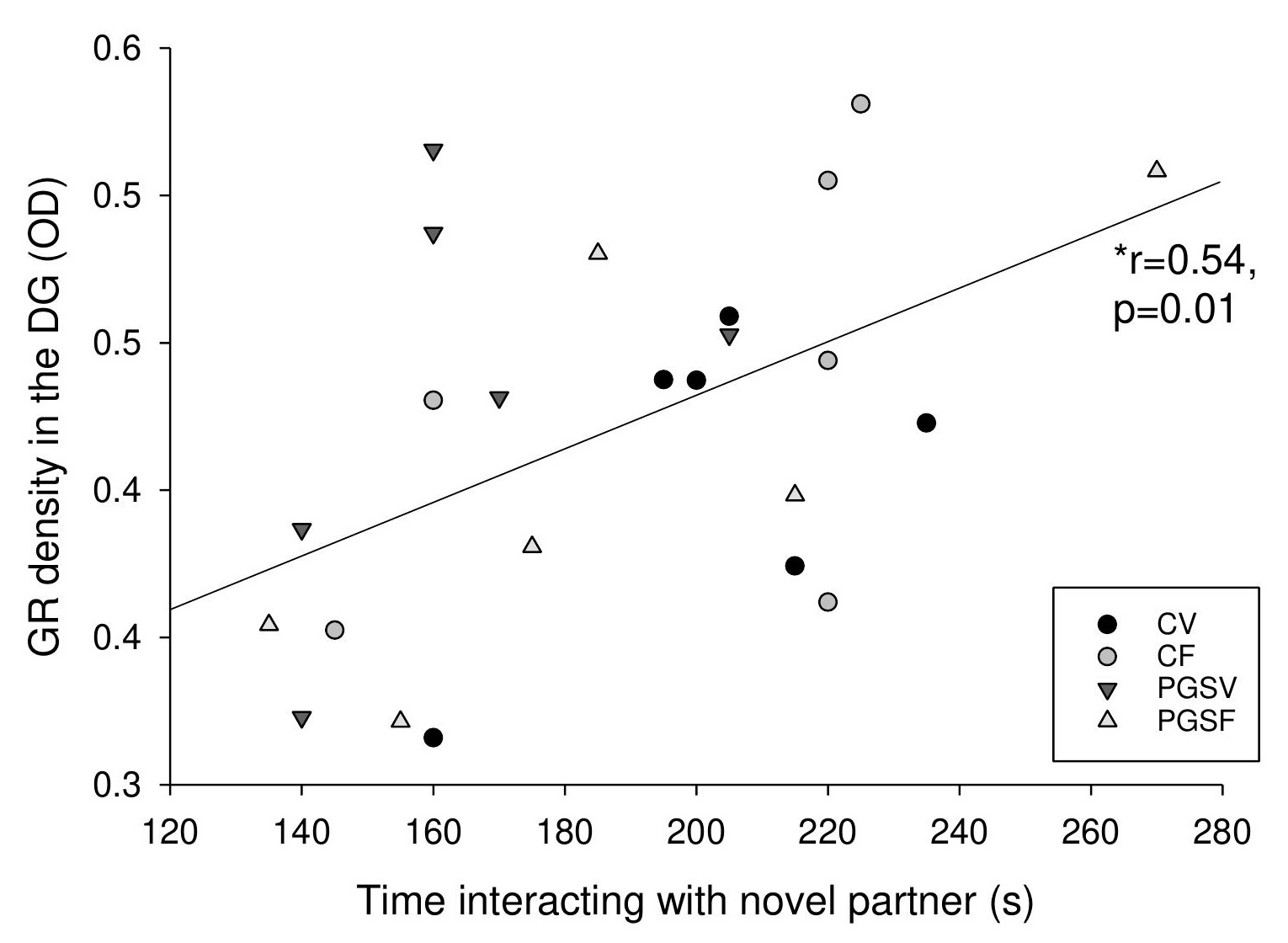


Figure 4

Female

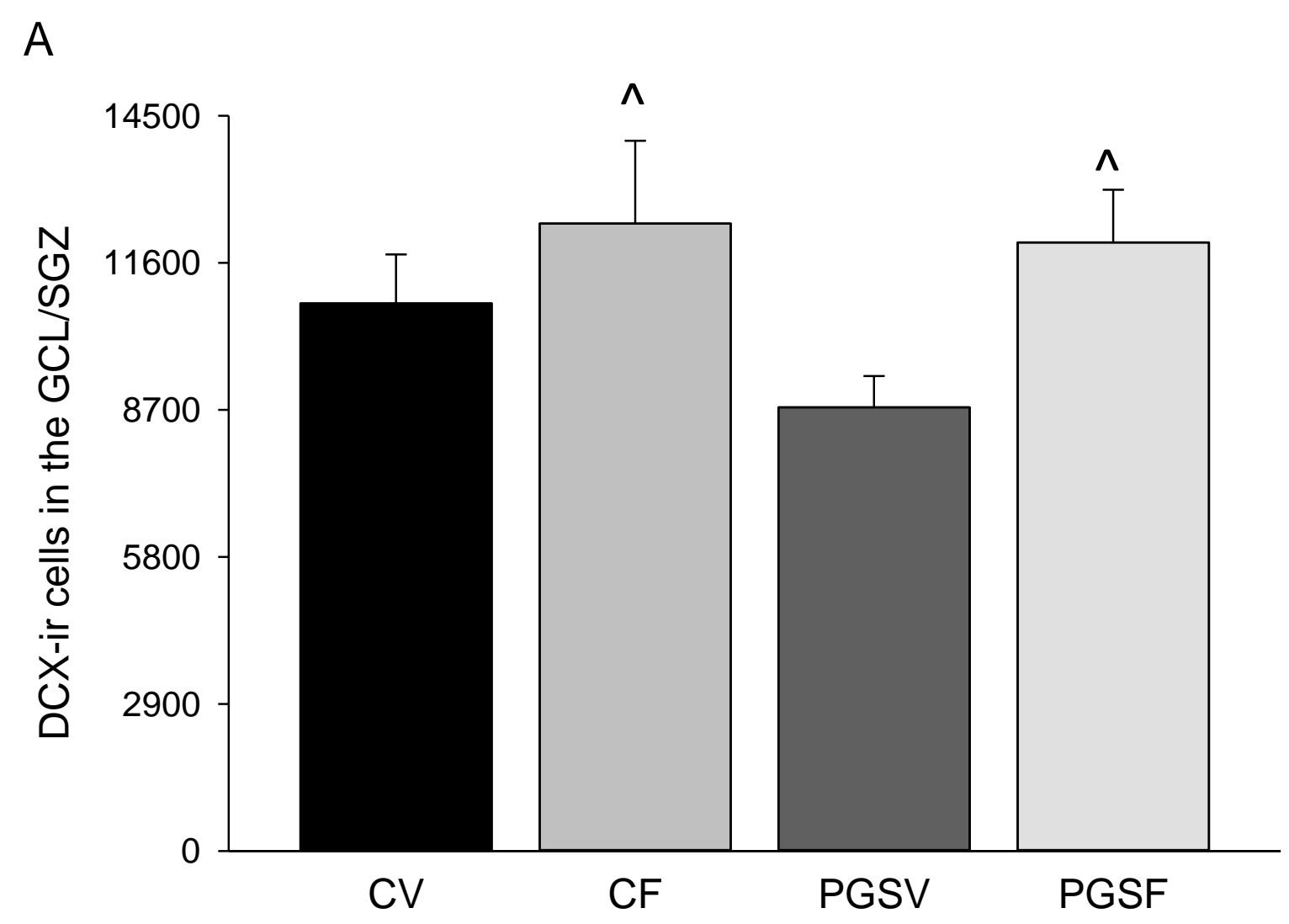

B

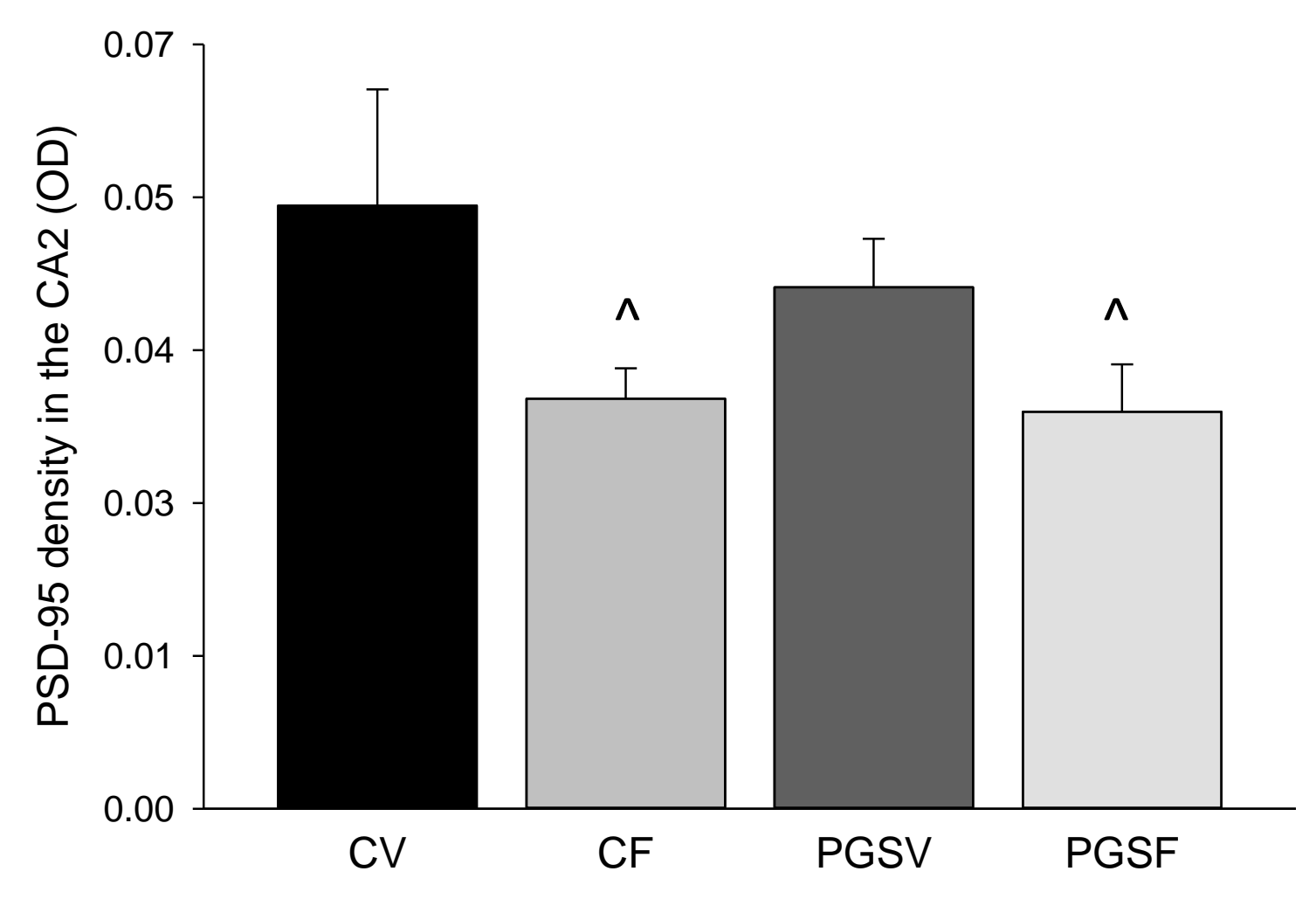

Male

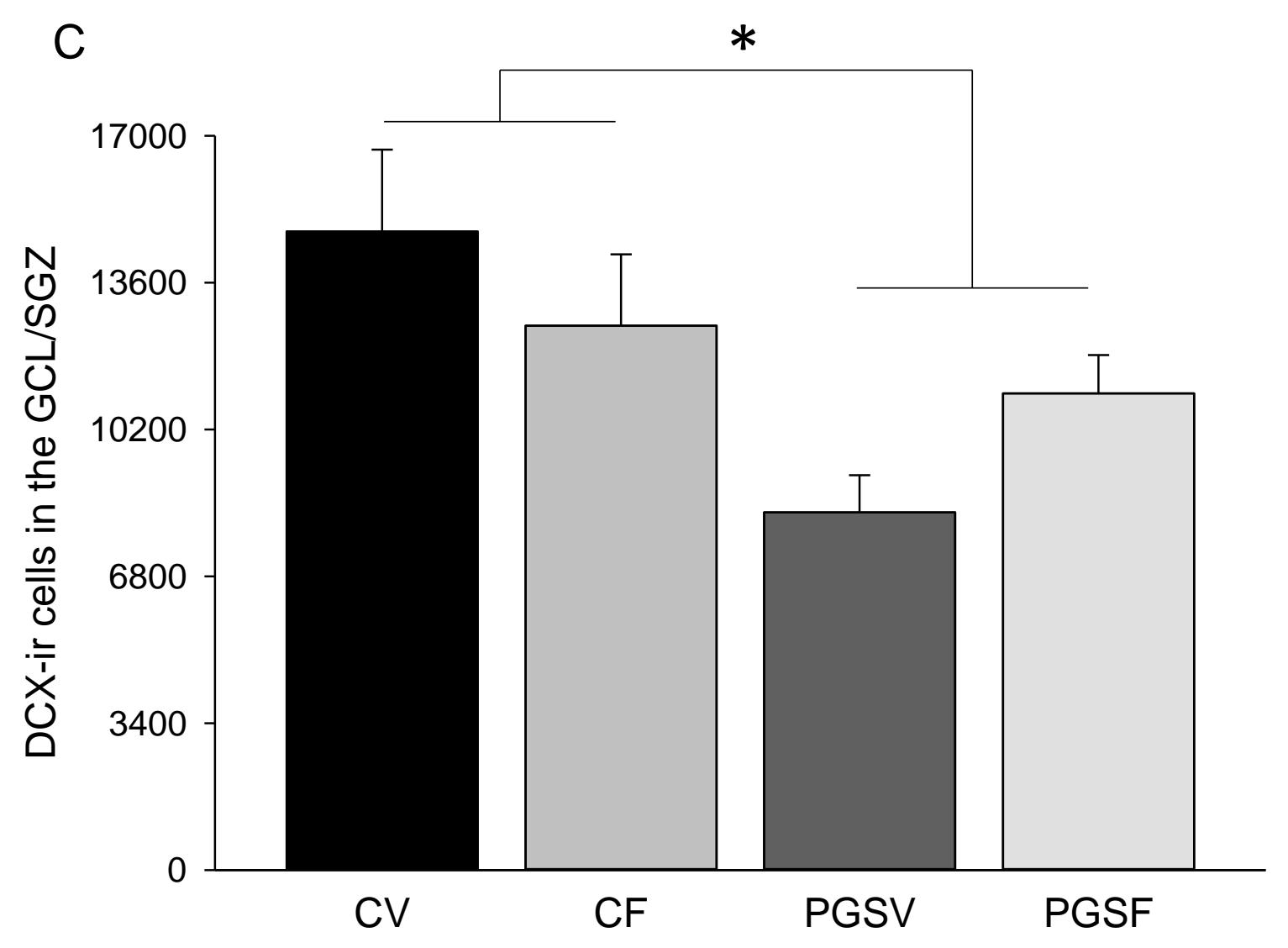

D

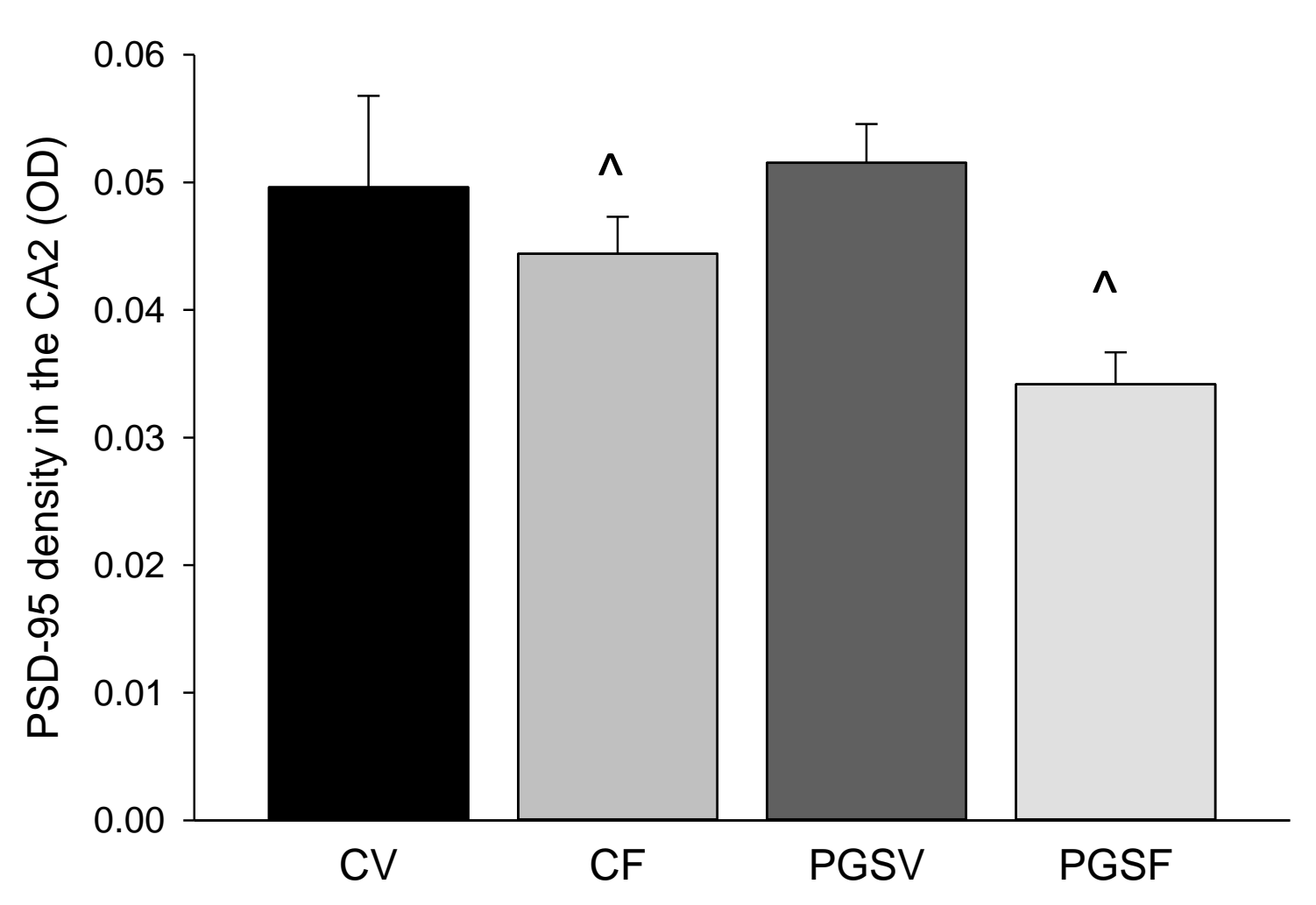




\section{Highlights}

- Perinatal SSRIs increase social investigation in females and social play in males

- Pre-gestational maternal stress affects social investigation in adult males only

- Perinatal SSRIs have sexually differentiated effects on hippocampal plasticity

- Perinatal SSRIs and maternal stress affect GR density in the hippocampus

- Fetal-sex specific treatment should be considered for maternal mental illness 